\title{
Synthetic Evaluation of MicroRNA-1-3p Expression in Head and Neck Squamous Cell Carcinoma Based on Microarray Chips and MicroRNA Sequencing
}

\author{
Yubing Chen, ${ }^{1}$ Mingjiang Liu, ${ }^{1}$ Hu Jin, ${ }^{1}$ Bo Peng, ${ }^{1}$ Luo Dai, ${ }^{1}$ Sifan Wang, ${ }^{1}$ Hao Xing, \\ Baoju Wang $\mathbb{D}^{2}$, and Zhan $W u \mathbb{D}^{1}$ \\ ${ }^{1}$ Division of Hepatobiliary Surgery, The First Affliated Hospital of Guangxi Medical University, Nanning, Guangxi, China \\ ${ }^{2}$ Department of Pathology, Xiangyang Central Hospital, Xiangcheng District, Xiangyang, Hubei, China
}

Correspondence should be addressed to Baoju Wang; 584963886@qq.com and Zhan Wu; wuzhan@stu.gxmu.edu.cn

Baoju Wang and Zhan Wu contributed equally to this work.

Received 24 May 2021; Accepted 9 August 2021; Published 25 August 2021

Academic Editor: Paul Harrison

Copyright (C) 2021 Yubing Chen et al. This is an open access article distributed under the Creative Commons Attribution License, which permits unrestricted use, distribution, and reproduction in any medium, provided the original work is properly cited.

Background. MicroRNA-1-3p (miR-1-3p) exerts significant regulation in various tumor cells, but its molecular mechanisms in head and neck squamous cell carcinoma (HNSCC) are still ill defined. This study is aimed at detecting the expression of miR1-3p in HNSCC and at determining its significant regulatory pathways. Methods. Data were obtained from the Cancer Genome Atlas (TCGA), Gene Expression Omnibus (GEO), Oncomine, ArrayExpress, Sequence Read Archive (SRA) databases, and additional literature. Expression values of miR-1-3p in HNSCC were analyzed comprehensively. The R language software was employed to screen differentially expressed genes, and bioinformatics assessment was performed. One sequence dataset (HNSCC: $n=484$; noncancer: $n=44$ ) and 18 chip datasets (HNSCC: $n=656$; noncancer: $n=199$ ) were obtained. Results. The expression of miR-1-3p in HNSCC was visibly decreased in compare with noncancerous tissues. There were distinct differences in tumor state $(P=0.0417)$, pathological stage $(P=0.0058)$, and T stage $(P=0.0044)$. Comprehensive analysis of sequence and chip data also indicated that miR-1-3p was lowly expressed in HNSCC. The diagnostic performance of miR-1-3p in HNSCC is reflected in the sensitivity and specificity of the collection, etc. Bioinformatics analysis showed the possible biological process, cellular component, molecular function, and KEGG pathways of miR-1-3p in HNSCC. And ITGB4 was a possible target of miR1-3p.Conclusions. miR-1-3p's low expression may facilitate tumorigenesis and evolution in HNSCC through signaling pathways. ITGB4 may be a key gene in targeting pathways but still needs verification through in vitro experiments.

\section{Introduction}

HNSCC is a human malignant neoplasm common in certain regions. The morbidity of HNSCC has improved dramatically in recent years, particularly among women. Each year, over 600,000 patients are diagnosed HNSCC globally, over half in the Asia-pacific regions [1]. Platinum-based chemotherapy, radiotherapy, and cetuximab are commonly used to treat recurrent/metastatic HNSCC in Asia [2]. Unfortunately, about $75 \%$ of these patients have local progression $(60 \%)$ or metastasis (15\%) upon first contact, and the median survival of patients with relapse and/or metastasis is only 6 months. Survival rates drop rapidly in patients who fail first-line treatment, often dying within three months [3]. Seeking antitumor therapies that are more effective, more scholars are paying close attention to moleculartargeted therapy. Recently, many studies on microRNAs (miRNAs) have been affirmed in the cancer field, and molecular-targeted therapy has become a burgeoning treatment for tumors [4].

MicroRNAs of approximately 22 nucleotides long are noncoding single-stranded RNAs, coded efficiently by endogenous genes [5]. They regulate the expression of posttranscription genes. Many studies have presented distinctions in the 


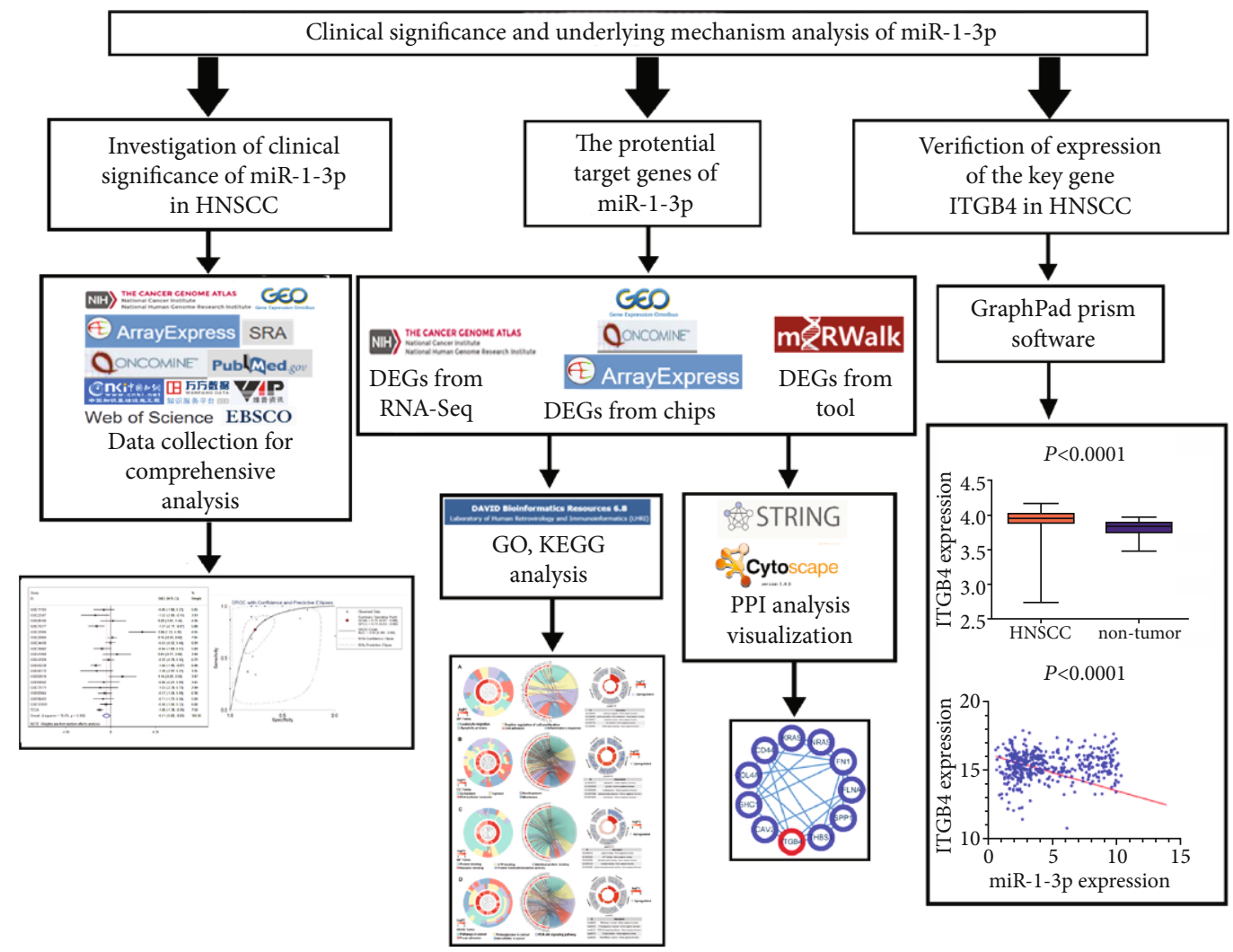

FIgURE 1: The research technical circuit diagram of this study.

expression of some miRNAs in cancerous and noncancerous tissues [6], utilizing signaling pathways to control key genes affecting physiological and biochemical processes such as proliferation and differentiation in tumor cells. Notwithstanding, the clinical value and molecular functions of individual miRNAs remain relatively unexplored. As a gene member, miRNA-1-3p targets different proteins or genes and affects the occurrence and development of gastric [7], colon [8], and breast carcinomas [9, 10], among others [11-13]. However, there are few relevant articles about the expression of miR-1-3p in HNSCC. Only 8 research teams have explored the ontology of miR-1-3p on key genes that regulate HNSCC $[1,14-20]$. Potential molecular mechanism regulation in HNSCC is still unclear, and new enrichment regulatory pathways need to be proposed.

In this study, a total of 1,140 cancer samples and 243 noncancer samples were collected based on sequencing, microarray, and literature data to probe the clinical significance and impact of miR-1-3p in HNSCC. Potential molecular mechanisms, including significant genes and enrichment paths of miR-1-3p in HNSCC, were summarized. ITGB4 was one of the target genes. The schematic of the research design is shown in Figure 1.

\section{Materials and Methods}

\subsection{Sources of miR-1-3p Expression Data in HNSCC}

2.1.1. MicroRNA-Seq Data. The miRNA sequence dataset was obtained from TCGA [21], including samples of cancerous and noncancerous tissues. The steps for data download were as follows: the site for UCSC-Xena (https://xena.ucsc.edu/) visited, checked "TCGA hub" in the "DATASETS" option, and selected "TCGA(HNSC)," which contains 25 datasets. In the new interface, selected "IlluminaHiseq $(n=529)$ TCGA hub" to download matrix files and gene annotation files. By matching the two, the expression value of miR-1-3p was determined. After deleting missing data, mature miR-1-3p expression data were extracted. Meanwhile, using "sangerbox," the corresponding clinical case data were downloaded for analysis.

2.1.2. Microarray Data. Microarrays were filtered to evaluate miR-1-3p expression in Gene Expression Omnibus (GEO) [22]. The overall strategy for retrieval was OSCC OR HNSCC OR "head and neck" OR "nasopharynx." We adjusted search terms to achieve the best range. The search was restricted to "Series" in "Entry type" and "H. sapiens" in "organisms." All research contained in the chips followed these criteria: (1) the species is human, (2) the objective is tissue, (3) microarray results include required gene, (4) containing both HNSCC tissues and nontumor tissues, and (5) miR-1-3p expression is mature. Conversely, the exclusion criteria were (1) objects other than human, (2) serum sample or other liquid types, (3) the chip does not contain the required genes, (4) there is only the experimental group or the control group, (5) control samples have other related diseases, and (6) include drug-related research or other unrelated interventions. After filtering the chips, chip number was input into the GEO database for querying detailed data; 


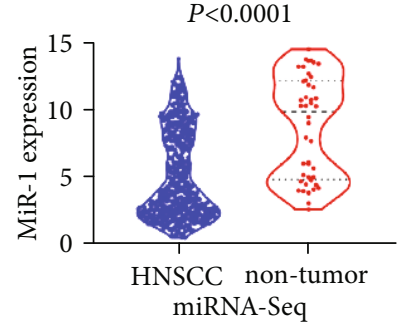

(a)

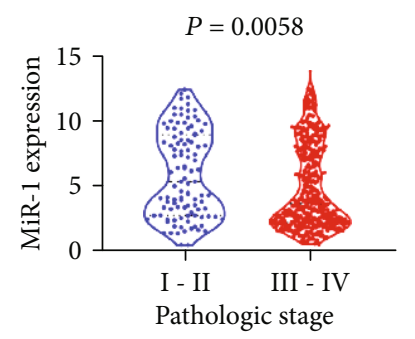

(c)

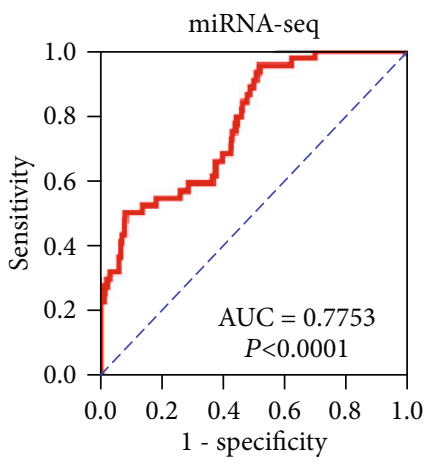

(e)

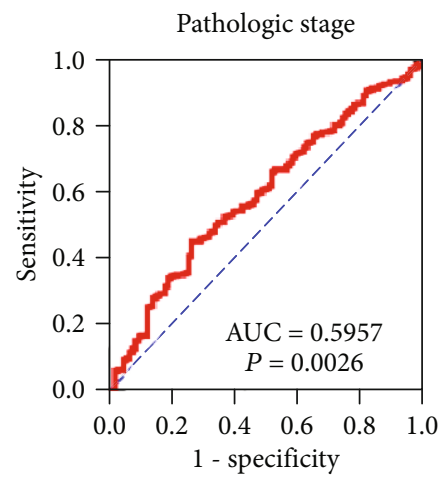

(g)

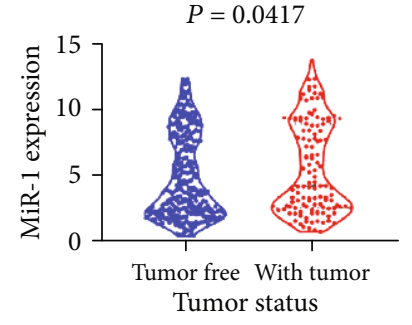

(b)

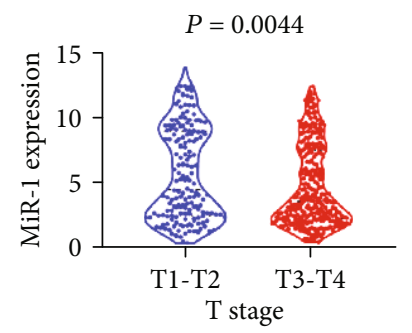

(d)

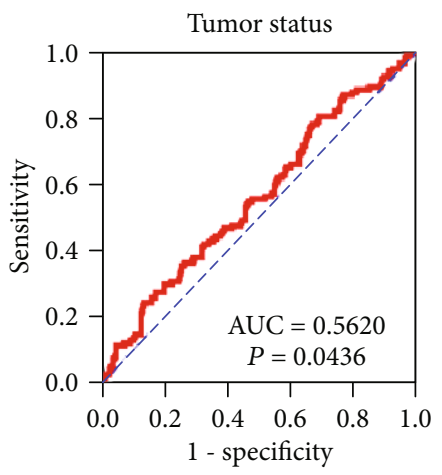

(f)

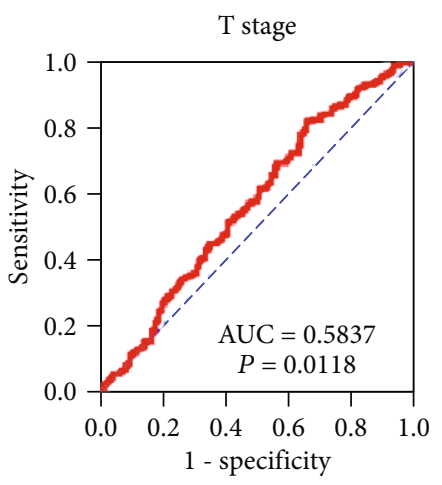

(h)

FIGURE 2: The miR-1-3p expression decreases in different clinicopathological parameters of HNSCC: (a-d) violin figure; (e-h) ROC curve. (a, e) The total expression of miR-1-3p in HNSCC and nontumor tissue from the TCGA database. (b, f) The relationship between miR-1-3p expression and tumor status. (c, g) The relationship between miR-1-3p expression and pathologic stage. (d, h) The relationship between miR-1-3p expression and T stage. The differential expression of miR-1-3p in HNSCC was statistically significant, which was manifested in tissue, tumor status, pathological stage, and T stage. AUC: the area under the ROC curve; $P$ value: $T$-test with two independent samples.

we downloaded the "SOFT formatted family file(s)" probe annotation files and "Series Matrix file(s)" gene probe expression Matrix file, to find the miR-1-3p corresponding expression data. For chip screening in ArrayExpress [23], Oncomine [24], and SRA [25], the methods were the same as above.
2.1.3. Literature Data. We reviewed articles about gene expression in HNSCC in Chinese and foreign scientific research sites, including CNKI, Wanfang, Vip, PubMed, Web of Science, and EBSCO databases. We extracted these chips to supplement existing microarrays to acquire unabridged data. 
2.2. Comprehensive and Detailed Analysis. Based on the sequencing data and the specific content of each microarray, the data were selected to make $\log 2$ processing or not. The mean expression level (mean) and standard deviation (SD) were calculated using SPSS 22.0. The "car" package of $\mathrm{R}$ was employed to draw violin plots to clarify that the miR-1-3p's expression was different in cancerous and noncancerous tissues. To evaluate the expression level of miR-1-3p comprehensively, a meta-analysis of continuous variables was conducted with Stata 12.0. When heterogeneity was small $\left(I^{2}<50 \%\right)$, the fixed effect model was adopted for analysis. On the contrary, if heterogeneity was extensive $\left(I^{2}>50 \%\right)$, a random-effect model was used, and we continued to perform sensitivity analysis to determine the sources of heterogeneity. After removing the chips that contributed to heterogeneity, we reanalyzed the results based on remaining data. If heterogeneity was less than $50 \%$, the results were reliable. For statistical analysis, if the standard mean deviation $(\mathrm{SMD})<0$ and the $95 \%$ confidence interval (CI) did not cross the 0 -point coordinate line, the gene was demonstrated to be significantly lower in HNSCC. An SMD > 0 meant that the research object was highly expressed in carcinoma. The ROC curves were plotted using SPSS, and the sROC curve was drawn using the Stata software to forecast the clinical significance of miR-1-3p in HNSCC. When the area under the curve was $>0.5$, this index had a certain diagnostic value for diseases. The AUC over 0.7 indicated a good diagnostic value. Publication bias was determined using Begg's funnel plot.

2.3. Screening of Differential Genes. Based on $\log |\mathrm{FC}|>1$ and $P<0.05,39$ arrays of the expression of long noncoding genes in HNSCC and $3 \mathrm{miR}-1-3 \mathrm{p}$ transfection samples (GSM610393, GSM610394, GSM639297) were selected from TCGA, GEO, Oncomine, and ArrayExpress databases. DEGs that appeared over 8 times were singled out from 39 datasets, combined with miR-1-3p transfected samples and the prediction tool (miRWalk 2.0) to form final differential genes of HNSCC.

2.4. Bioinformatics Analytical Methodology. Gene annotation and pathway enrichment analysis of DEGs were conducted in DAVID v 6.8; top-ranked annotations and pathways were listed, and $P<0.05$ indicated that the difference was statistically significant. The MCODE plugin in Cytoscape 3.4.0 is an APP that performs topological gathering on a fixed net to find dense connection areas. All differentially expressed genes enriched in five Kyoto Encyclopedia of Genes and Genomes (KEGG) pathways were introduced into the software. In addition, the most central modules in the PPI network were confirmed by MCODE.

2.5. Verify the Expression of Target Genes. Gene expression profile information of the 11 key genes in the sequence was imported into the cBioPortal website for genetic variation to verify the regulatory relationship between pivotal genes and miR-1-3p. In addition, the GraphPad Prism 8 software was used to verify the association between miR-1$3 p$ and key genes.
TABLE 1: The relationship between miR-1-3p gene expression and clinical parameters in TCGA by using $T$-test.

\begin{tabular}{|c|c|c|c|c|}
\hline Parameters & $N$ & Mean \pm SD & $T$ value & $P$ value \\
\hline \multicolumn{5}{|l|}{ Tissue } \\
\hline HNSCC & 484 & $5.139 \pm 3.274$ & 6.048 & 0.0001 \\
\hline Normal & 44 & $8.709 \pm 3.788$ & & \\
\hline \multicolumn{5}{|l|}{ Age } \\
\hline$\geq 60$ & 267 & $5.027 \pm 3.259$ & 0.756 & 0.4500 \\
\hline$<60$ & 214 & $5.253 \pm 3.275$ & & \\
\hline \multicolumn{5}{|l|}{ Gender } \\
\hline Male & 350 & $5.099 \pm 3.284$ & 0.465 & 0.6420 \\
\hline Female & 132 & $5.255 \pm 3.267$ & & \\
\hline \multicolumn{5}{|c|}{ Lymphovascular invasion } \\
\hline Yes & 113 & $5.897 \pm 3.548$ & 1.702 & 0.0905 \\
\hline No & 211 & $5.223 \pm 3.314$ & & \\
\hline \multicolumn{5}{|l|}{ Tumor status } \\
\hline With tumor & 124 & $5.649 \pm 3.467$ & 1.967 & 0.0417 \\
\hline Tumor free & 310 & $4.941 \pm 3.177$ & & \\
\hline \multicolumn{5}{|c|}{ Histological grade } \\
\hline G3-G4 & 122 & $5.307 \pm 3.487$ & 0.382 & 0.7022 \\
\hline G1-G2 & 340 & $5.175 \pm 3.204$ & & \\
\hline \multicolumn{5}{|l|}{ Pathologic stage } \\
\hline III-IV & 376 & $4.924 \pm 3.239$ & 2.771 & 0.0058 \\
\hline I-II & 106 & $5.915 \pm 3.308$ & & \\
\hline \multicolumn{5}{|l|}{$\mathrm{T}$ stage } \\
\hline T3-T4 & 299 & $4.814 \pm 3.069$ & 2.762 & 0.0044 \\
\hline $\mathrm{T} 1-\mathrm{T} 2$ & 172 & $5.702 \pm 3.515$ & & \\
\hline \multicolumn{5}{|l|}{$\mathrm{N}$ stage } \\
\hline N1-N3 & 239 & $5.055 \pm 3.268$ & 0.763 & 0.4465 \\
\hline No & 225 & $5.287 \pm 3.274$ & & \\
\hline \multicolumn{5}{|l|}{ M stage } \\
\hline M1 & 5 & $2.968 \pm 1.602$ & 3.043 & 0.0541 \\
\hline M0 & 457 & $5.198 \pm 3.279$ & & \\
\hline \multicolumn{5}{|l|}{ Margin status } \\
\hline Positive & 55 & $5.246 \pm 3.219$ & 0.457 & 0.6480 \\
\hline Negative & 322 & $5.467 \pm 3.344$ & & \\
\hline
\end{tabular}

\section{Results}

3.1. miR-1-3p Expression Decreased in HNSCC in miRNASeq. In total, 44 nontumor and 484 HNSCC tissue samples were included in the miRNA sequence. Compared with noncancerous tissues, the expression of miR-1-3p in HNSCC visibly decreased $(5.139 \pm 3.275$ vs. $8.709 \pm 3.788, P<0.001)$ (Figure 2(a)). The area under the ROC curve (AUC) was $0.775(P<0.001)$ (Figure $2(\mathrm{e})$ ). From the statistical analysis of clinical parameters, the expression of miR-1-3p in tumor tissues showed distinct differences in tumor state $(P=0.0417$, Figure $2(\mathrm{~b}))$, pathological stage $(P=0.0058$, Figure $2(\mathrm{c}))$, and T stage $(P=0.0044$, Figure $2(d))$ (Table 1$)$. 
TABLE 2: The gene chip dataset information of miR-1-3p.

\begin{tabular}{|c|c|c|c|c|c|}
\hline Chip name & First author & Country & Public year & Sample & Platform \\
\hline GSE11163 & Michele Avissar & USA & 2008 & Tissue & GPL6690 \\
\hline GSE22587 & Yang Shu & China & 2013 & Tissue & GPL8933 \\
\hline GSE28100 & Hyunmin Jung & USA & 2012 & Tissue & GPL1085 \\
\hline GSE31277 & Patricia Severino & Brazil & 2014 & Tissue & GPL4133 \\
\hline GSE32906 & Zhaohui Luo & China & 2012 & Tissue & GPL11350 \\
\hline GSE32960 & Jun Ma & China & 2012 & Tissue & GPL14722 \\
\hline GSE34496 & Michael F Ochs & USA & 2013 & Tissue & GPL8786 \\
\hline GSE36682 & Rongrong Wei & China & 2012 & Tissue & GPL15311 \\
\hline GSE41268 & Zijun Xie & China & 2012 & Tissue & GPL10850 \\
\hline GSE43329 & Jinze Zheng & China & 2013 & Tissue & GPL16475 \\
\hline GSE45238 & Shine-Gwo Shiah & China & 2015 & Tissue & GPL8179 \\
\hline GSE46172 & Jeffrey Bethony & USA & 2014 & Tissue & GPL16770 \\
\hline GSE62819 & Jugao Fang & China & 2014 & Tissue & GPL16384 \\
\hline GSE69002 & Chad Creighton & USA & 2016 & Tissue & GPL18044 \\
\hline GSE73171 & Zenghong Li & China & 2016 & Tissue & GPL14613 \\
\hline GSE82064 & Nicola Valeri & Switzerland & 2017 & Tissue & GPL21968 \\
\hline GSE98463 & Cintia Micaela Chamorro & Spain & 2017 & Tissue & GPL21572 \\
\hline GSE10393 & Yujin Hoshida & USA & 2017 & Tissue & GPL3921 \\
\hline
\end{tabular}

TABLE 3: Basic statistical indicators of miR-1-3p expression values in the experimental groups and control groups were summarized.

\begin{tabular}{lcccccccccc}
\hline Name & Case_n & Case_mean & Case_sd & Cont_n & Cont_mean & Cont_sd & TP & FP & FN & TN \\
\hline GSE11163 & 16 & 5.0060 & 2.9502 & 5 & 6.9577 & 3.0365 & 11 & 1 & 5 & 4 \\
GSE22587 & 8 & 1.3000 & 5.5401 & 4 & 10.1731 & 6.4789 & 7 & 1 & 1 & 3 \\
GSE28100 & 17 & 9.1189 & 2.1971 & 3 & 8.6146 & 3.0807 & 15 & 2 & 2 & 1 \\
GSE31277 & 15 & 3.4416 & 0.2865 & 15 & 3.7763 & 0.1914 & 13 & 5 & 2 & 10 \\
GSE32906 & 16 & 7.7991 & 2.3468 & 6 & 1.2160 & 1.4288 & 6 & 1 & 10 & 5 \\
GSE32960 & 312 & 8.6397 & 0.3116 & 18 & 8.5930 & 0.3889 & 211 & 8 & 101 & 10 \\
GSE34496 & 44 & 1.2115 & 0.3470 & 25 & 1.2232 & 0.3832 & 15 & 6 & 29 & 19 \\
GSE36682 & 62 & 8.7352 & 0.4357 & 6 & 9.0868 & 0.0713 & 54 & 0 & 8 \\
GSE41268 & 7 & -1.1088 & 3.6207 & 3 & -3.0293 & 0.3337 & 2 & 0 & 5 \\
GSE43329 & 31 & 6.5909 & 0.2343 & 19 & 6.6317 & 0.0071 & 17 & 4 & 14 & 15 \\
GSE45238 & 40 & 8.6857 & 2.4182 & 40 & 11.9760 & 2.0615 & 36 & 8 & 4 & 32 \\
GSE46172 & 4 & -2.8090 & 0.5321 & 4 & 3.9720 & 6.9307 & 3 & 0 & 1 \\
GSE62819 & 5 & 4.2859 & 3.0773 & 5 & 3.7886 & 3.0131 & 5 & 4 & 0 \\
GSE69002 & 3 & 2.9315 & 0.0689 & 4 & 2.9969 & 0.1152 & 3 & 2 & 0 \\
GSE73171 & 3 & 1.5082 & 0.0336 & 3 & 1.8233 & 0.4318 & 3 & 1 \\
GSE82064 & 35 & 5.9177 & 1.9298 & 12 & 6.9437 & 1.2942 & 14 & 0 \\
GSE98463 & 8 & 1.8867 & 1.9029 & 8 & 2.0199 & 2.4693 & 8 & 7 \\
GSE103931 & 30 & 5.0757 & 1.3494 & 19 & 5.9368 & 2.4772 & 29 & 14 & 0 \\
\hline
\end{tabular}

${ }^{a}$ Case_n, Case_mean, Case_sd: number, mean, standard deviation of experimental groups; Cont_n, Cont_mean, Cont_sd: number, mean, standard deviation of control groups; TP, FP, FN, TN: true positive, false positive, false negative, and true negative.

3.2. Expression and ROC Curves of $m i R-1-3 p$ in HNSCC in Different Gene Chips. Eighteen pieces of qualified chips were filtered from GEO, ArrayExpress, Oncomine, and SRA databases (Table 2). We calculated the mean and the SD in microarrays (Table 3) and plotted violin diagrams (Figure 3). We used adoptive data to calculate the AUC
(Figure 4). Foregoing chip results revealed that the miR-1$3 \mathrm{p}$ expression level in HNSCC was lower than in noncancer cells, in accordance with the results from the sequence.

3.3. Meta-analysis of miR-1-3p Expression Levels in HNSCC Decreased Compared to Nontumor Tissues. Detailed and 

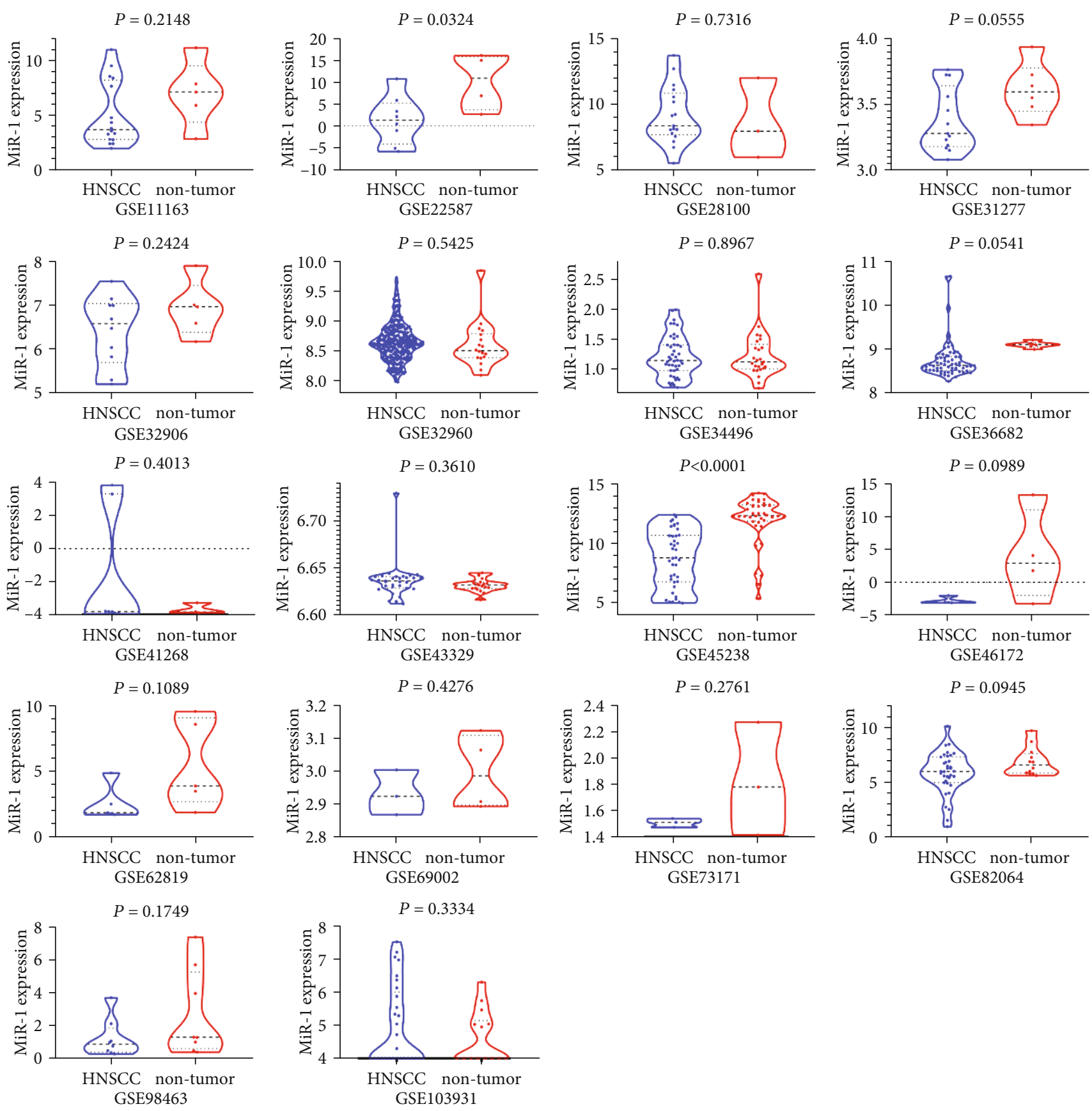

Figure 3: Expression of miR-1-3p in head and neck squamous cell carcinoma and noncancerous tissues in different gene chips.

comprehensive statistical analyses of data from the sequence and chips were performed to calculate the SMD values and to draw the forest plot $\left(\mathrm{SMD}=-0.59(-0.75,-0.43), I^{2}=\right.$ $78.5 \%, P<0.001$, Figure 5(a)). Data heterogeneity was overt. The random-effect model was used (Figure 5(b)) $\left(\mathrm{SMD}=-0.41(-0.80,-0.03), I^{2}=78.5 \%, P<0.001\right)$. In the figure, the diamond occupied left of the invalid vertical line, miR-1-3p was weakly expressed in HNSCC, and the difference was statistically significant. Further sensitivity analysis was performed (Figure 5(c)). We selected four most influential studies to detect possible sources of heterogeneity
(Figure 5(d)). Results showed that $I^{2}=47.0 \%, P=0.023$, heterogeneity decreased obviously, and the difference was statistically significant. This heterogeneity might come from these four chips: GSE32906, GSE32960, GSE34496, and GSE45238. The European SMD value was -0.28 (95\% CI: $-0.60,0.04)\left(I^{2}=0.0 \%\right)$, and the Asian SMD value was -0.32 (95\% CI: $-0.92,0.28)\left(I^{2}=83.7 \%\right)$ (Figure 5(e)), suggesting that heterogeneity may be derived from the country subgroup. The result of Begg's test was $P=0.961$, indicating that there was no apparent publication bias in our study (Figure 5(f)). 

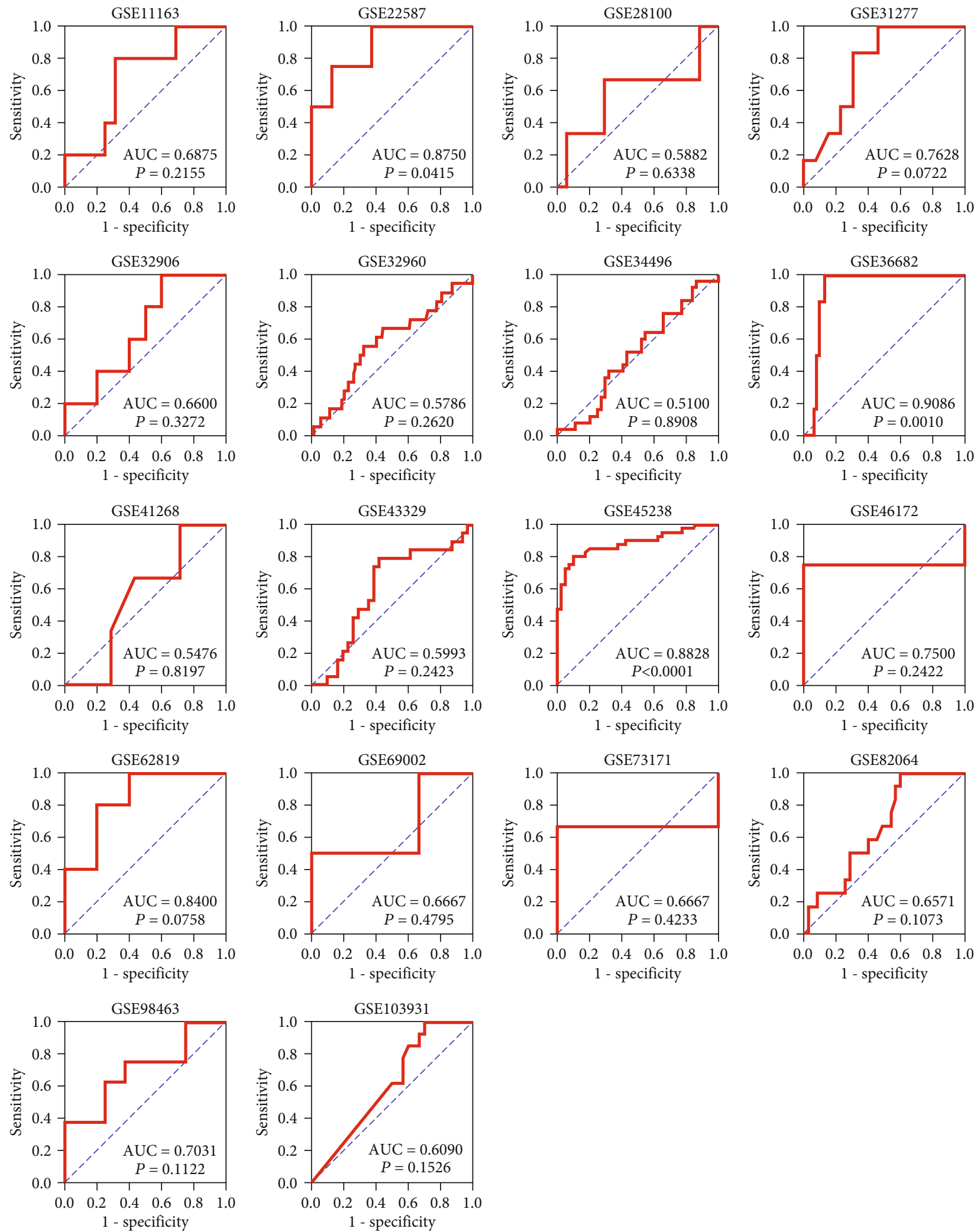

FIGURE 4: ROC curves of miR-1-3p in HNSCC and nontumor tissues in different gene chips.

3.4. Clinical Significance and Value of miR-1-3p in HNSCC. We computed the true positive (TP), false positive (FP), false negative $(\mathrm{FN})$, and true negative (TN) of each dataset based on the most approximate den index and the corresponding cutoff value (Table 3 ). The area under the sROC curve was 0.83 (95\% CI: 0.80-0.86) (Figure 6(a)). The values of total 


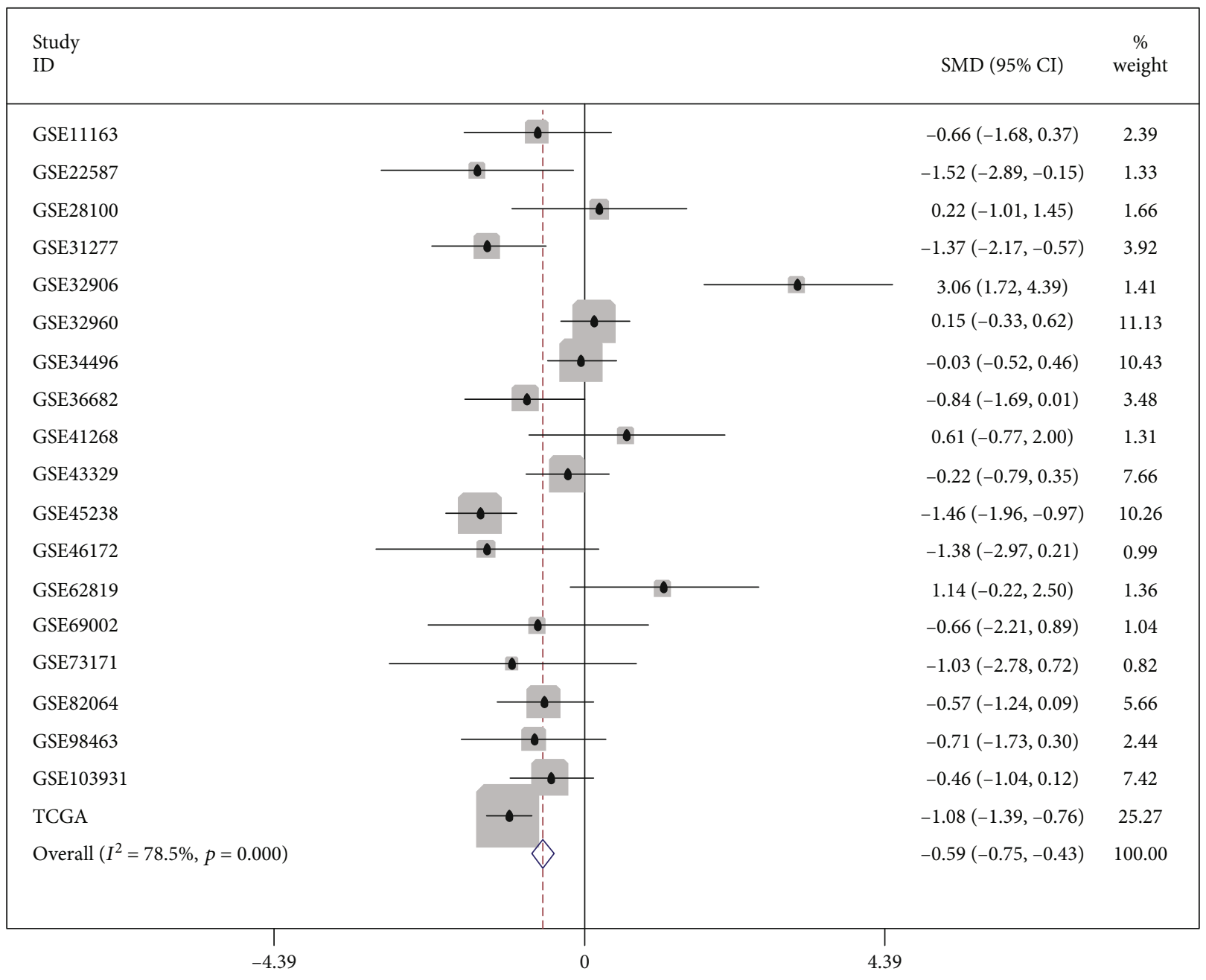

(a)

Figure 5: Continued. 


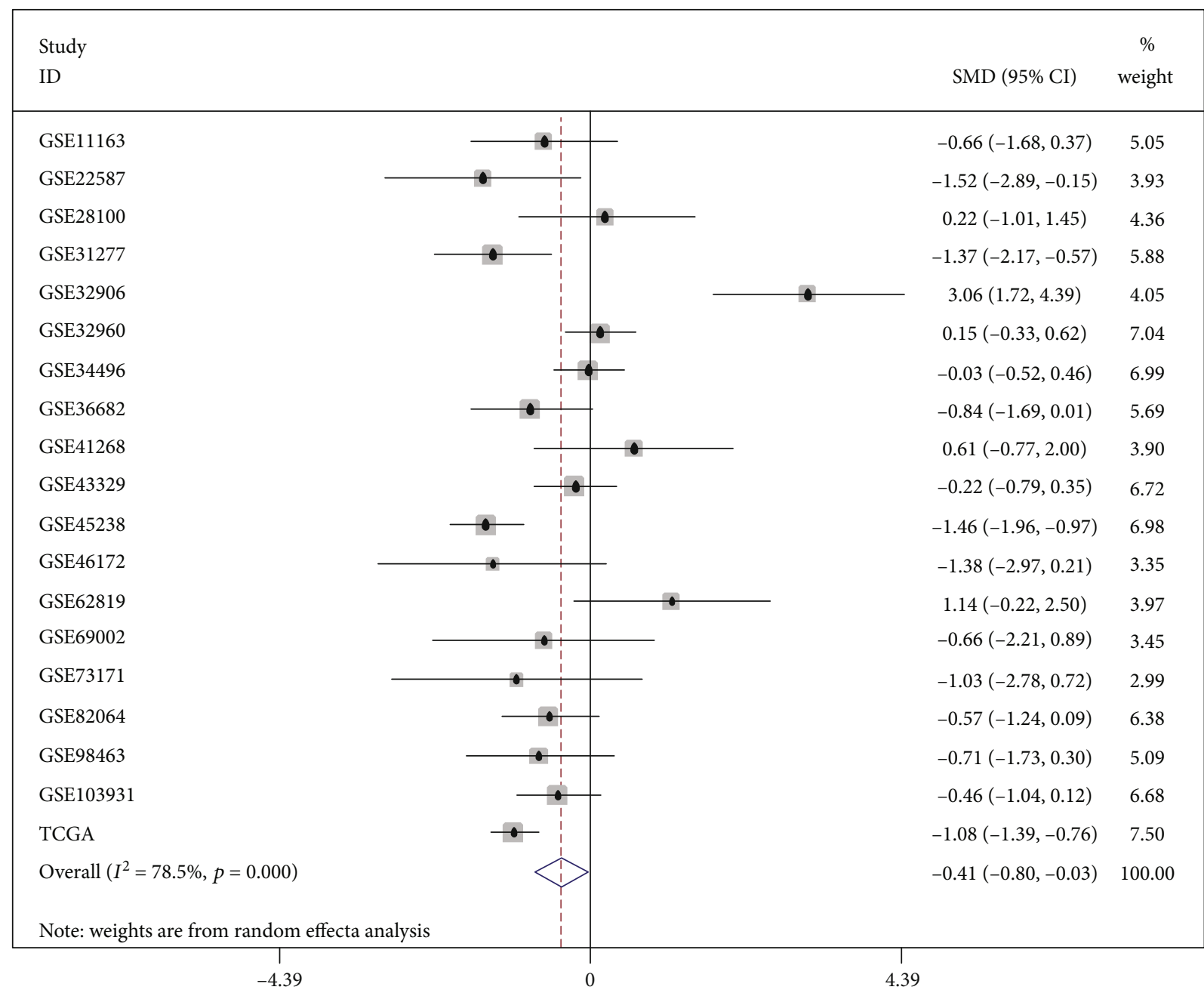

(b)

Figure 5: Continued. 


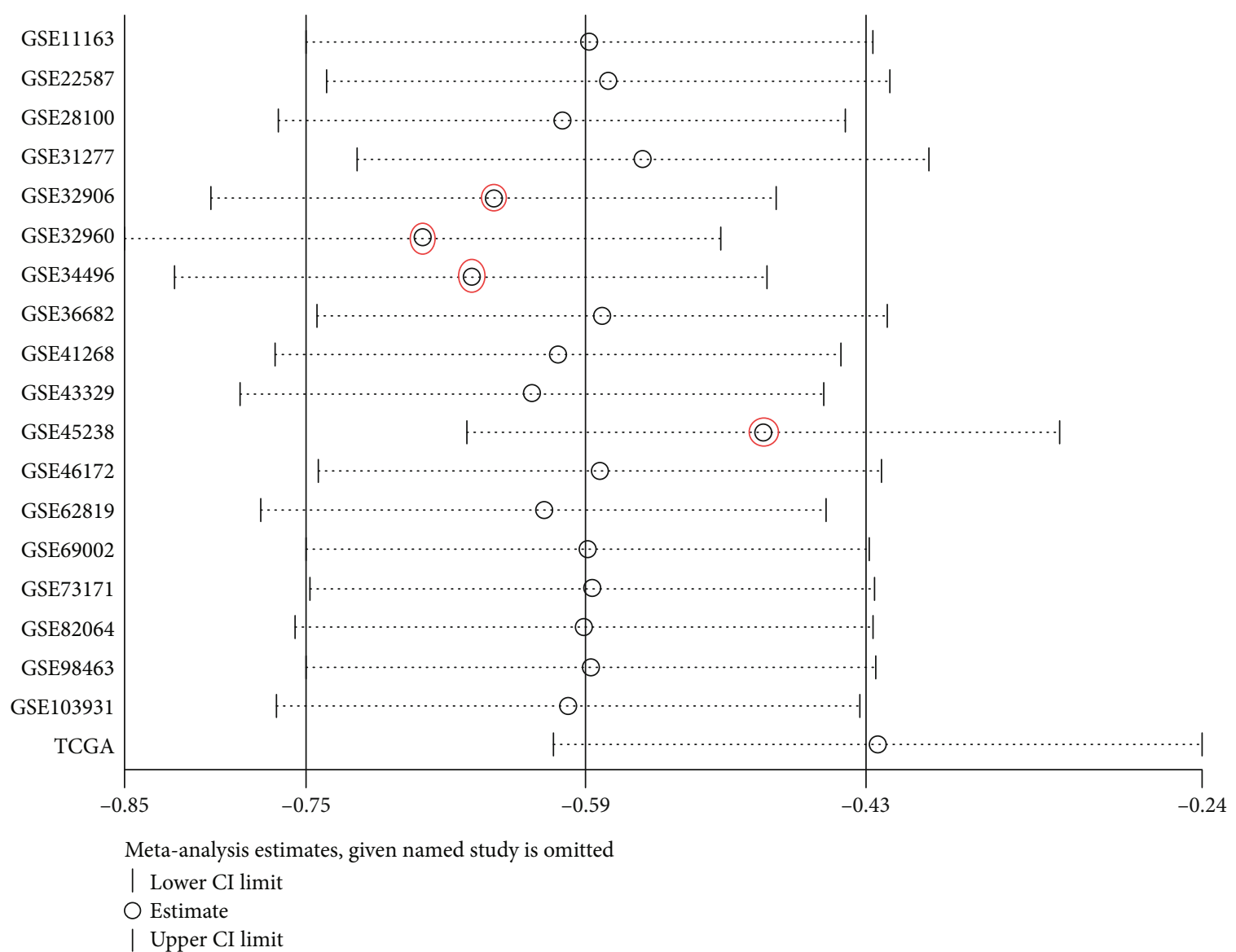

(c)

FIgURe 5: Continued. 


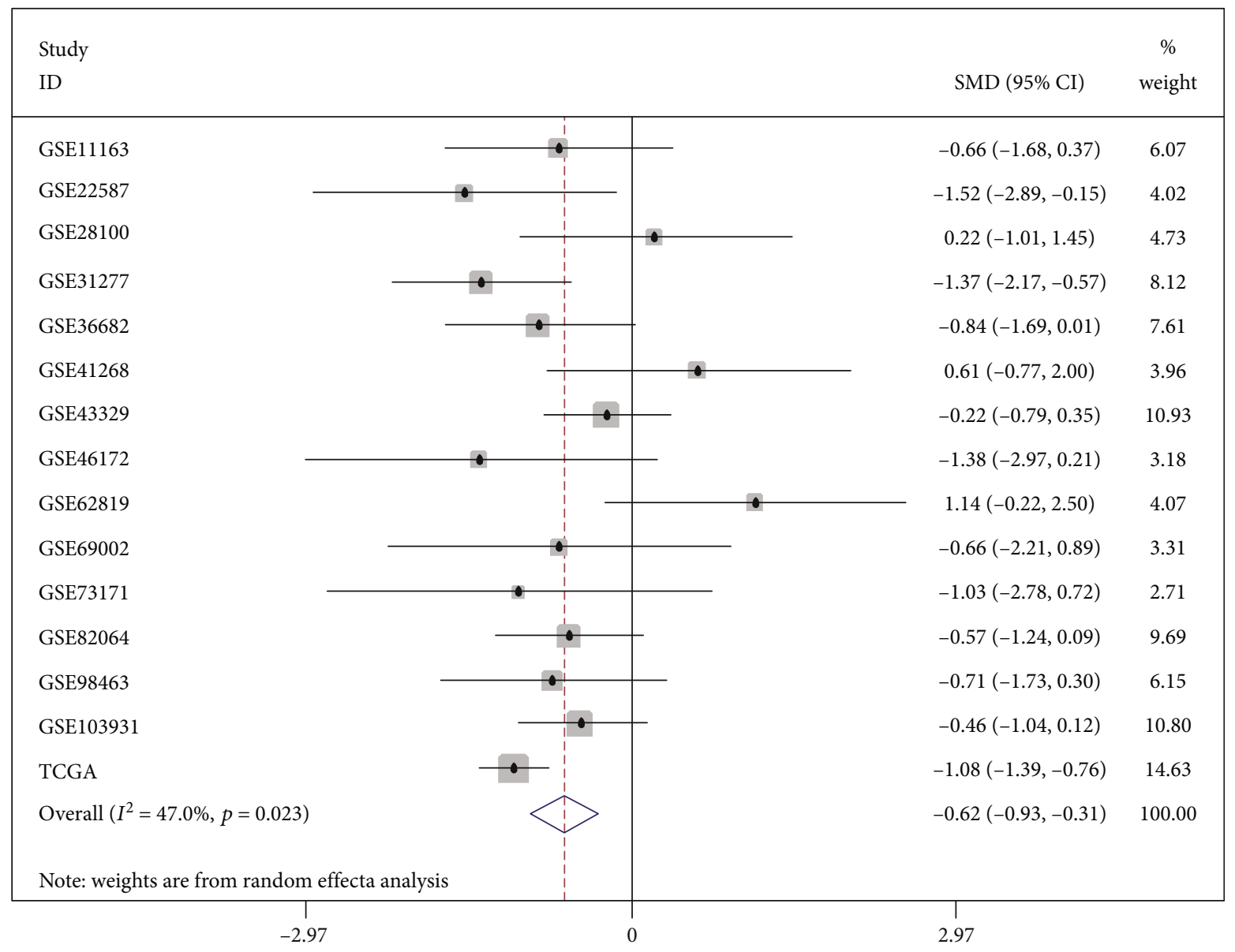

(d)

FIgUre 5: Continued. 


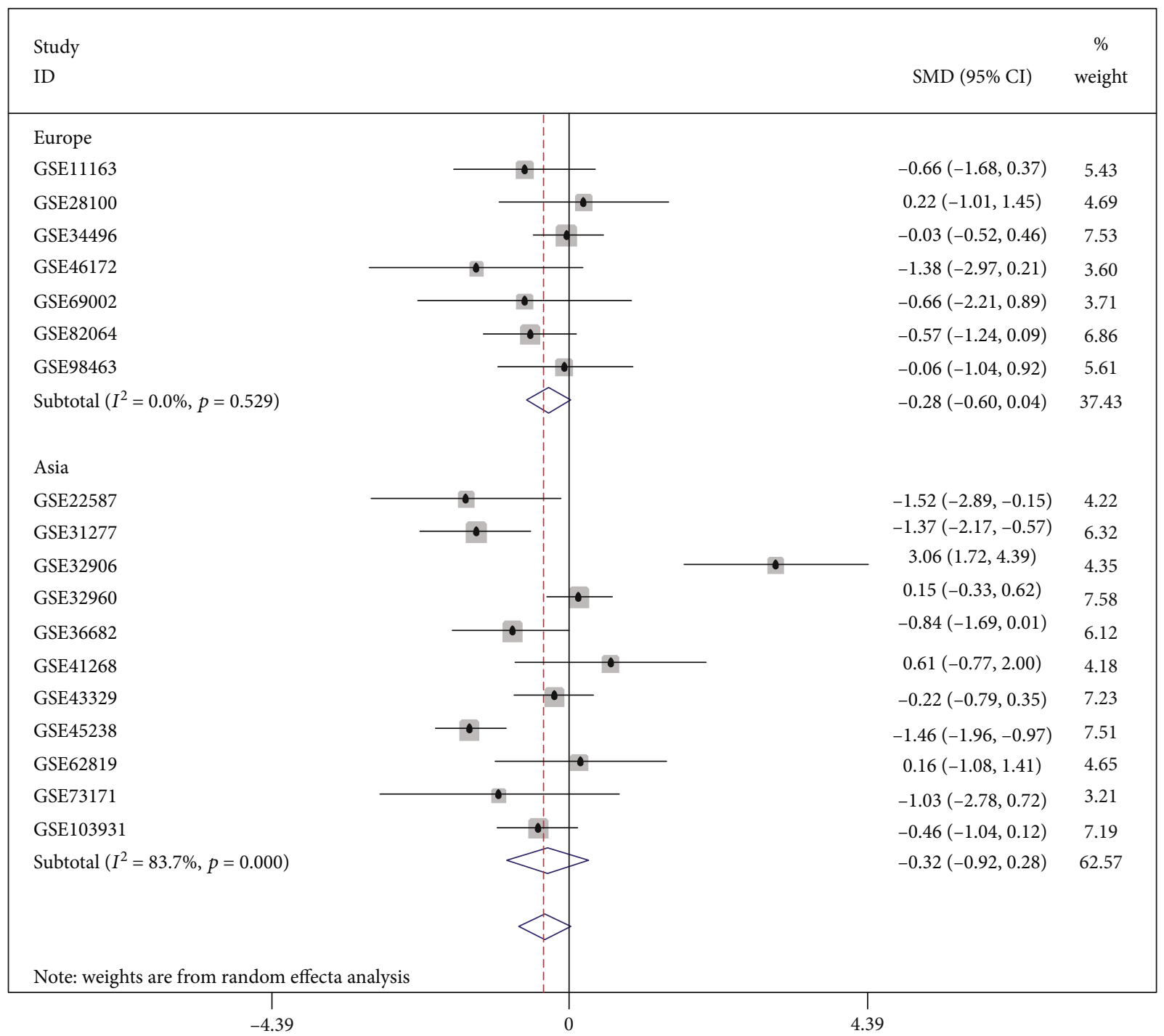

(e)

Figure 5: Continued. 


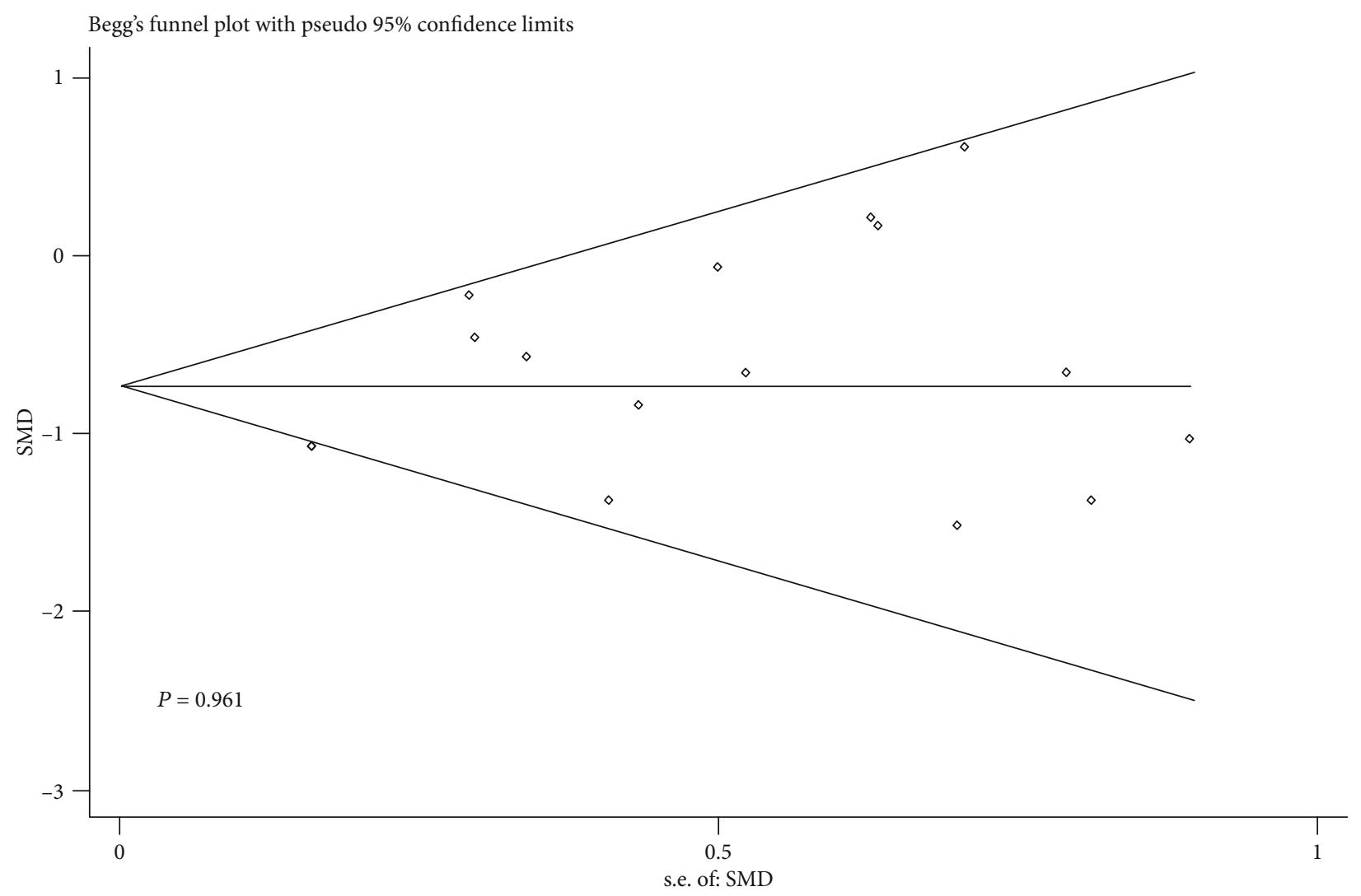

(f)

Figure 5: The meta-analysis of miR-1-3p expression levels in HNSCC decreases compared to nontumor tissues. (a) Forest map of SMD (fixed-effect model). (b) Forest plot of SMD (random-effect model). (c) The sensitivity analysis. (d) After the heterogeneity studies were eliminated, the forest plot of SMD based on 15 microarrays. (e) Subgroup analysis of countries was carried out to further explore the sources of heterogeneity. (f) Begg's funnel plot showed no obvious publication bias.

sensitivity, total specificity, positive likelihood ratio (PLR), negative likelihood ratio (NLR), diagnosis rate (DOR), and 95\% confidence interval were 0.41 (95\% CI: $0.38-0.44)$ (Figure 6(b)), 0.77 (95\% CI: 0.72-0.82) (Figure 6(c)), 2.31 (95\% CI: 1.56-3.40) (Figure 6(d)), 0.40 (95\% CI: 0.26-0.61) (Figure 6(e)), and 7.87 (95\% CI: 4.22-14.69) (Figure 6(f)), respectively.

3.5. Gene Enrichment Analysis of miR-1-3p in HNSCC. According to the $\log \mathrm{FC}$ and $P$ value, 174 upregulated differentially expressed genes and 103 downregulated genes were screened. Given that miR-1-3p was lowly expressed in HNSCC, we chose 174 upregulated DEGs for GO annotation and KEGG enrichment by DAVID 6.8 $(P<0.05)$, whose top five enriched terms were summarized according to $P$ value (Table 4 ). Leukocyte migration, positive regulation of cell proliferation, apoptotic process, cell adhesion, and inflammatory response were the five most conspicuous terms for biological process (BP). In the cellular component (CC), genes were major enriched in the cytoplasm, cytosol, nucleoplasm, extracellular exosome, and membrane. As for molecular function $(\mathrm{MF})$, the coexpressed proteins were involved in protein binding, ATP binding, identical protein binding, receptor binding, and protein heterodimerization activity. For the KEGG pathway, the coexpressed genes were major gathered in pathways in cancer, proteoglycans in cancer, PI3K-Akt signaling pathway, focal adhesion, and microRNAs in cancer (Figure 7). In these genes, gene networks showed that KRAS, CD44, COL4A1, SHC1, CAV2, ITGB4, THBS1, SPP1, FLNA, FN1, and NRAS were closely connected in the pathways (Figure $8(\mathrm{a})$ ).

3.6. Preliminary Prediction of ITGB4 as Target Gene of $m i R-1-3 p$. The results of cBioPortal showed that the 11 target genes had different degrees of variation in HNSCC, reflected in a missense mutation, gene fusion, gene amplification, and gene deletion (Figure 8(b)). Only 2 HNSCC articles mentioned ITGB4 and showed that it may be the target of HNSCC, which can promote distant metastasis of tumors through the blood. Pearson correlation analysis showed that miR-1-3p had a correlation with ITGB4, which was statistically significant $(P<0.001)$ (Figure $8(c)$ ). For this reason, this gene was selected for further verification, and special attention was focused on the expression and prognosis of ITGB4 in HNSCC (Figures 8(d) and $8(\mathrm{e}))$. 


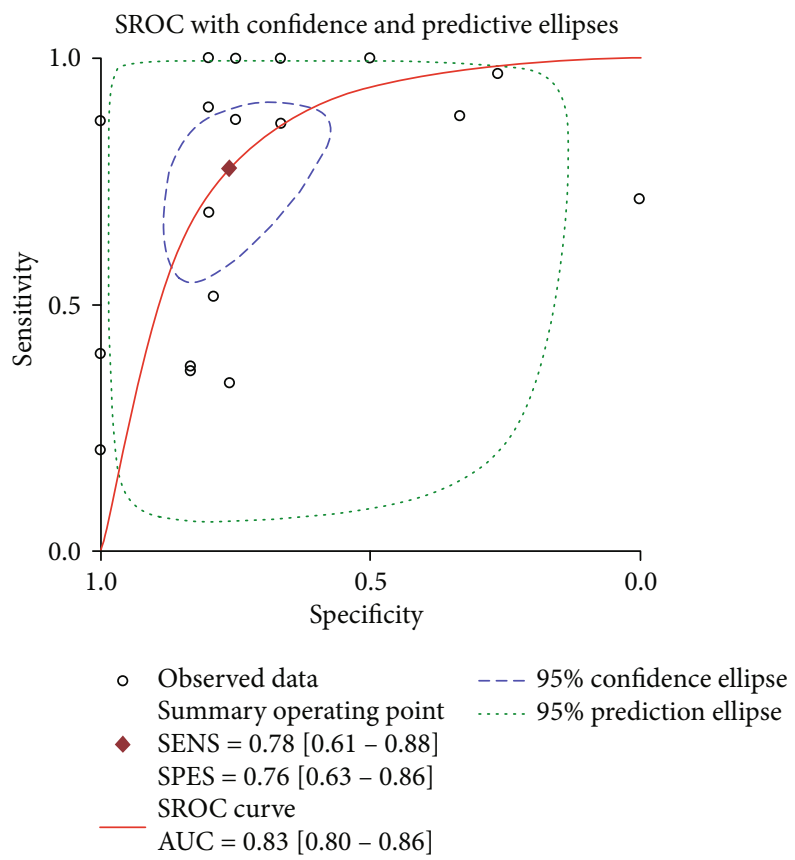

(a)

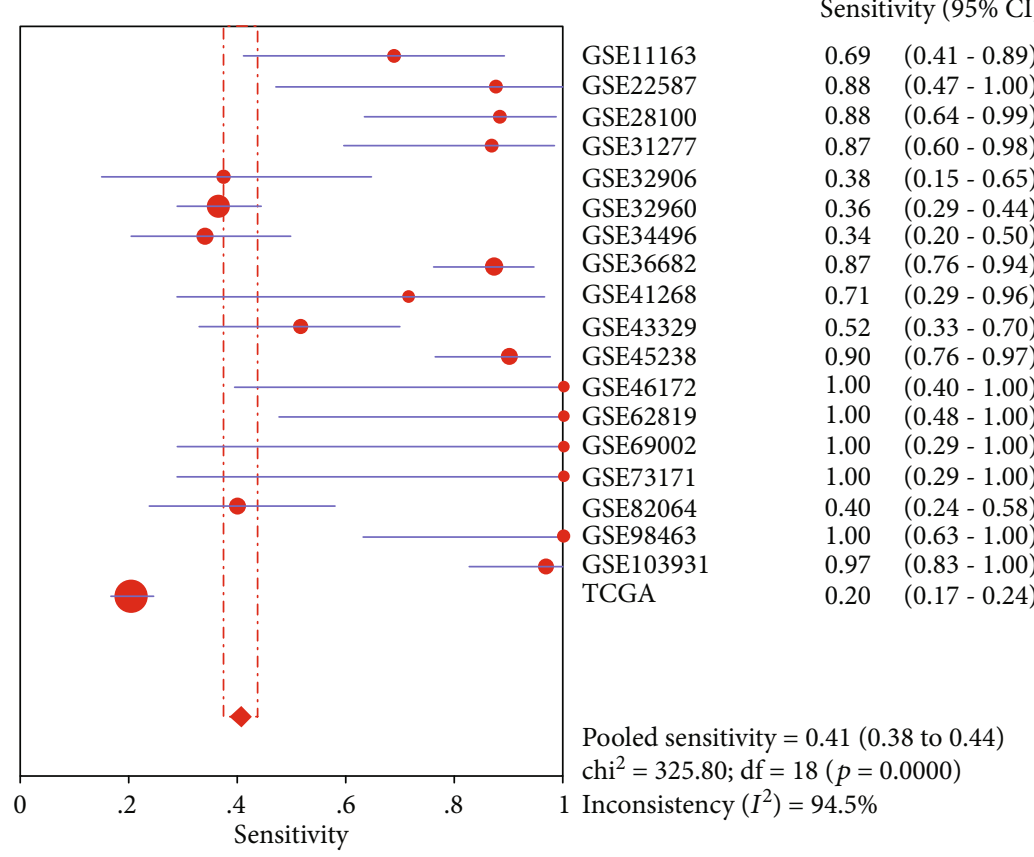

(b)

Figure 6: Continued. 


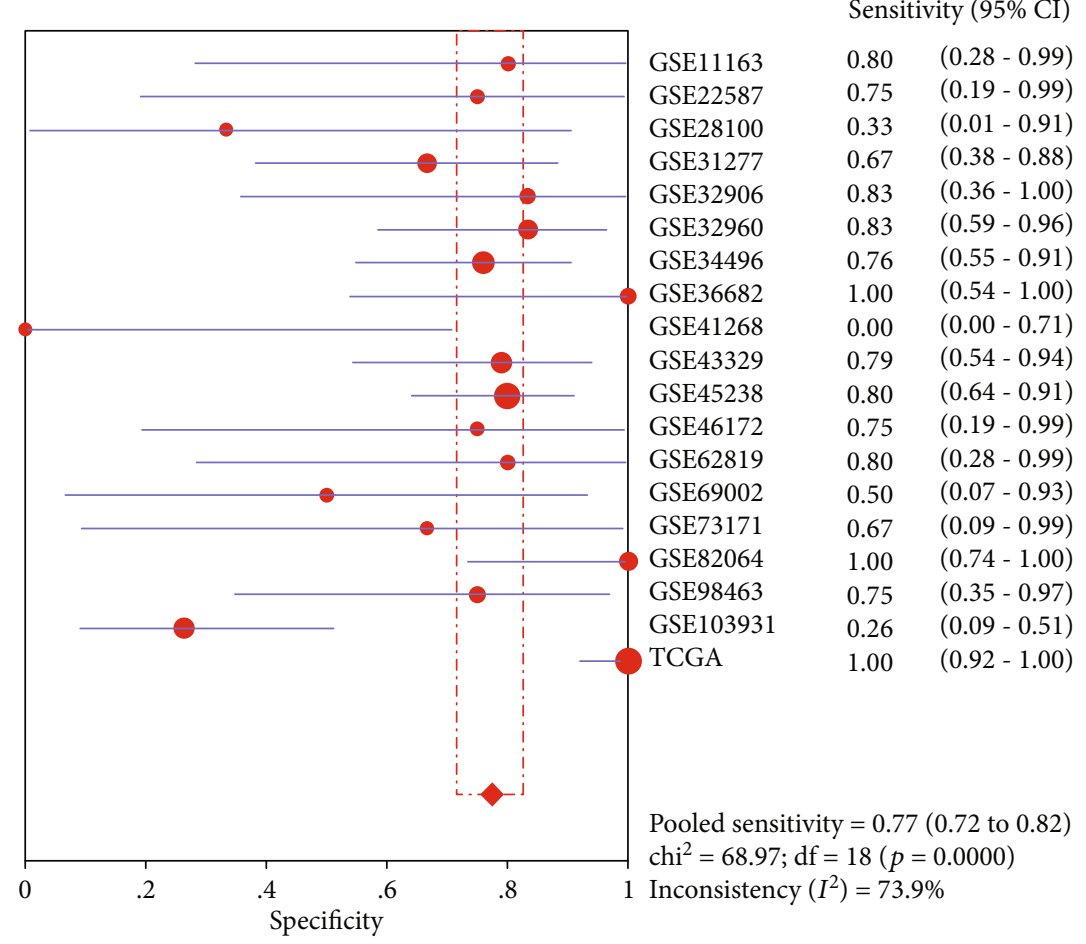

(c)

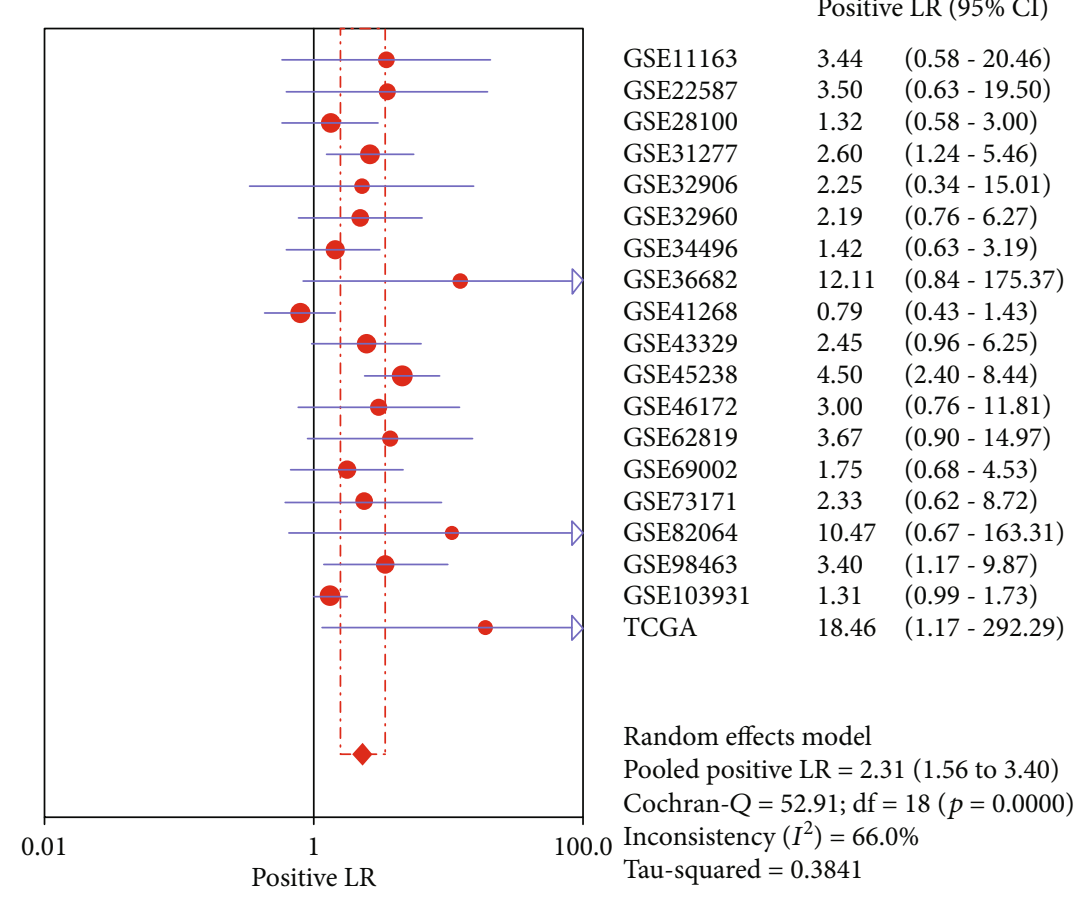

(d)

FIgUre 6: Continued. 


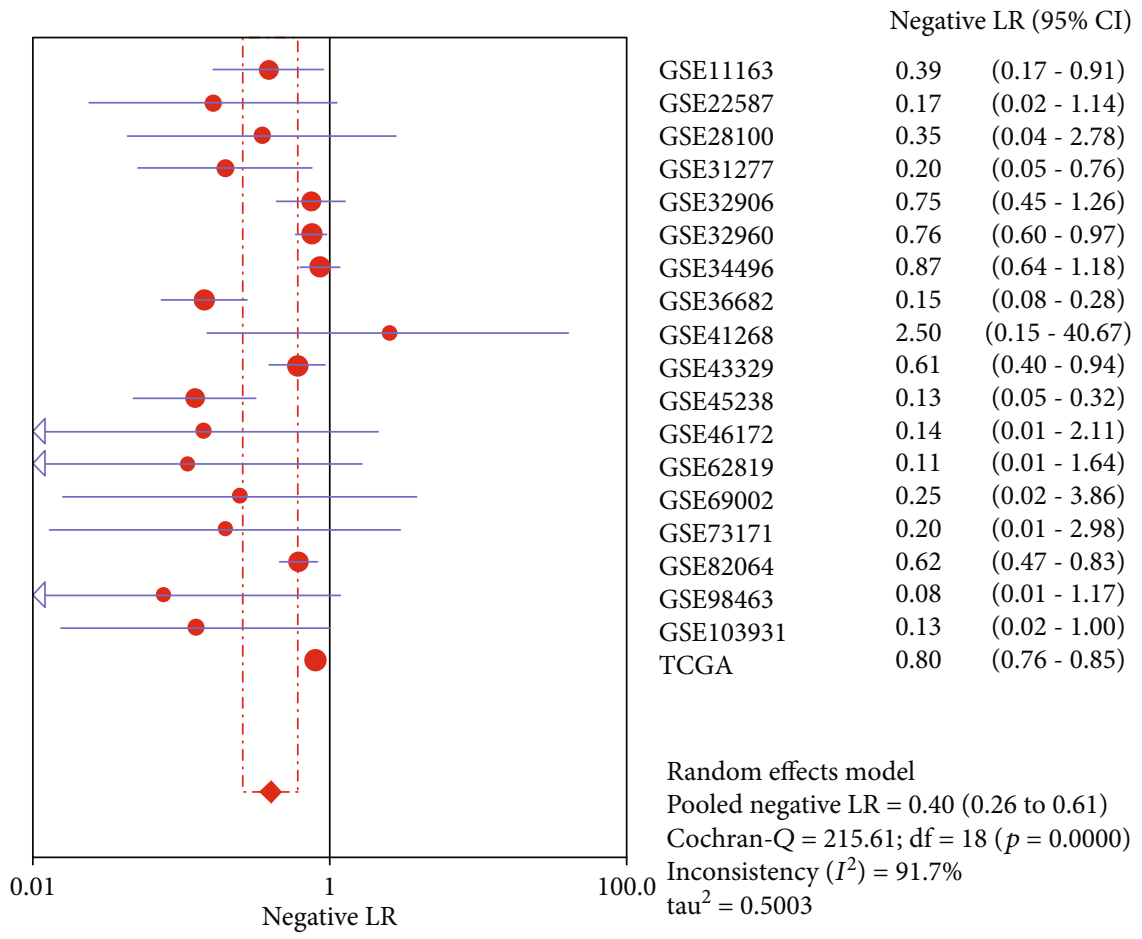

(e)

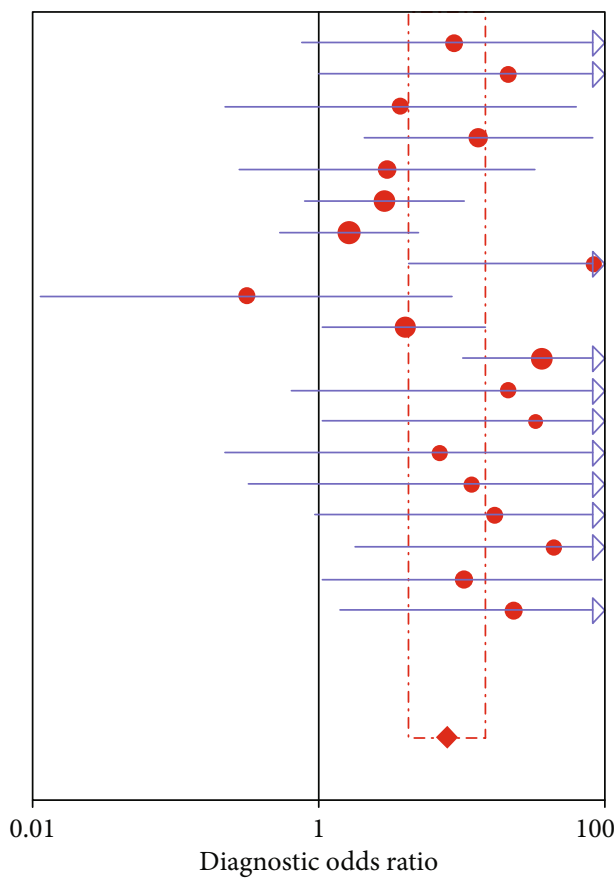

Diagnostic OR (95\% CI)

GSE11163

$8.80 \quad(0.77-100.26)$

GSE22587

GSE28100

GSE31277

GSE32906

GSE32960

GSE34496

GSE36682

GSE41268

GSE43329

GSE45238

GSE46172

GSE62819

GSE69002

GSE73171

GSE82064

$21.00(0.96-458.85)$

$3.75 \quad(0.22-62.76)$

$13.00(2.07-81.48)$

$3.00 \quad(0.28-32.21)$

$2.87 \quad(0.80-10.34)$

$1.64(0.54-4.97)$

$83.35(4.29-1,618.01)$

$0.31 \quad(0.01-8.68)$

$4.00 \quad(1.08-14.81)$

$36.00(9.90-130.94)$

$21.00(0.64-689.99)$

$33.00(1.06-1,023.57)$

$7.00 \quad(0.22-218.95)$

$11.67(0.32-422.14)$

$16.86(0.92-307.69)$

$44.20(1.80-1,088.15)$

$\begin{array}{lll}\text { GSE103931 } & 10.36(1.10-97.27) \\ \text { TCGA } & 22.97(1.40-376.27)\end{array}$

TCGA

$22.97(1.40-376.27)$

Random effects model

Pooled diagnostic odds ratio $=7.87$ (4.22 to 14.69$)$

Cochran- $\mathrm{Q}=26.77 ; \mathrm{df}=18(p=0.0834)$

Inconsistency $\left(I^{2}\right)=32.8 \%$

$\operatorname{tau}^{2}=0.5556$

(f)

Figure 6: The values of total sensitivity, total specificity, positive likelihood ratio (PLR), negative likelihood ratio (NLR), diagnosis rate (DOR), and 95\% confidence interval are statistically significant. (a) The SROC curve of miR-1-3p expression based on 19 datasets. (b-f) The forest map showed the diagnostic performance of miR-1-3p in HNSCC: the sensitivity of the collection, the specificity of the collection, the positive likelihood ratio of the summary, the negative likelihood ratio of the summary, and the summary diagnostic ratio based on the qualified dataset. 
TABLE 4: Gene ontology analysis of DEGs involved in biological process, cellular component, molecular function, and KEGG pathways.

\begin{tabular}{|c|c|c|c|c|c|}
\hline Category & & Term & Genes & Count & $P$ value \\
\hline \multirow{5}{*}{$\begin{array}{l}\text { Biological } \\
\text { process }\end{array}$} & GO:0050900 & Leukocyte migration & $\begin{array}{l}\text { CD44, KRAS, LYN, SHC1, CAV1, FN1, ITGB1, } \\
\text { MIF, MMP1, MSN, MYH9, NRAS, OLR1, } \\
\text { SLC7A11, SLC7A5, SLC7A8 }\end{array}$ & 16 & $4.40 E-13$ \\
\hline & GO:0008284 & $\begin{array}{l}\text { Positive regulation } \\
\text { of cell proliferation }\end{array}$ & $\begin{array}{c}\text { E2F3, FOSL1, KRAS, LYN, SHC1, TTK, ADM, } \\
\text { BIRC5, CSNK2A1, FN1, ITGB1, IL24, OSMR, } \\
\text { PGF, THBS1, TNFSF4 }\end{array}$ & 16 & $3.00 E-05$ \\
\hline & GO:0006915 & Apoptotic process & $\begin{array}{l}\text { BAX, BCL2L11, HTATIP2, BIRC5, CSNK2A1, } \\
\text { CLIC4, HIP1, IGFBP3, IL1A, IL1B, IL2RA, } \\
\text { IL24, PLSCR1, PHLDA2, STAT1, SULF1 }\end{array}$ & 16 & $2.60 E-04$ \\
\hline & GO:0007155 & Cell adhesion & $\begin{array}{c}\text { ABL2, CD44, CASK, DST, FN1, LOXL2, } \\
\text { NRP2, OLR1, PXN, SPP1, THBS1, TROAP }\end{array}$ & 12 & $3.80 E-03$ \\
\hline & GO:0006954 & $\begin{array}{l}\text { Inflammatory } \\
\text { response }\end{array}$ & $\begin{array}{l}\text { CXCL3, LYN, NMI, IL1A, IL1B, IL2RA, IL24, } \\
\text { MIF, OLR1, SPP1, THBS1 }\end{array}$ & 11 & $2.90 E-03$ \\
\hline \multirow{5}{*}{$\begin{array}{l}\text { Cellular } \\
\text { component }\end{array}$} & GO:0005737 & Cytoplasm & $\begin{array}{c}\text { BAX, CD44, CDC42BPA, CDC42EP3, POLD1, } \\
\text { E2F3, ERCC6L, FRMD4A, FGD6, GNA13, GINS4, } \\
\text { HAUS2, HTATIP2, KRAS, LYN, NMI, NUDCD1, } \\
\text { OIP5, PDLIM7, PPFIA1, RAD54B, RECQL, TTK, } \\
\text { WDHD1, ADM, BIRC5, B2M, CASK, CA2, } \\
\text { CAPRIN1, CENPE, CLIC4, CCNA2, CDKN3, } \\
\text { DTL, DNAH17, DST, EXO1, FTH1, FLNA, } \\
\text { GJB3, GMPS, HIP1, HPRT1, HIF1A, ITGB1, } \\
\text { MIF, MSN, MYO1B, MYO5A, MYH9, } \\
\text { NASP, PXN, PSPH, PLAT, PHLDA2, PHLDB2, } \\
\text { KCNS3, PCNA, PSMB9, RGS4, STAT1, SSH1, } \\
\text { SLC7A5, SLC7A8, TACC3, TCOF1, TROAP }\end{array}$ & 68 & $1.10 E-04$ \\
\hline & GO:0005829 & Cytosol & $\begin{array}{c}\text { ABL2, ATP6V1C1, BAX, BCL2L11, CDC42EP3, } \\
\text { ERCC6L, ERF, FOSL1, HAUS2, KRAS, LYN, PPFIA1, SHC1, } \\
\text { AP2M1, BIRC5, CASK, CA2, } \\
\text { CSNK2A1, CAPRIN1, CDCA3, CENPE, CENPL, } \\
\text { CENPN, CLIC4, CCNE2, DST, FTH1, FLNA, GLS, } \\
\text { GMPS, HPRT1, HIF1A, IL1A, IL1B, MYO5A, } \\
\text { MYH9, NDE1, PXN, PLSCR1, PSPH, PSMB9, RGS4, } \\
\text { STAT1, SNRPF, SNRPG, SLC7A5, TGM2, TPM3 }\end{array}$ & 48 & $2.20 E-04$ \\
\hline & GO:0005654 & Nucleoplasm & $\begin{array}{l}\text { POLD1, DSCC1, E2F3, ERCC6L, ERF, GABPB1, } \\
\text { GINS2, GINS4, HAUS2, HTATIP2, NMI, OIP5, } \\
\text { POP1, RAD54B, RAD54L, RECQL, WDHD1, } \\
\text { BIRC5, CSNK2A1, CENPL, CENPN, CCNA2, CCNE2, } \\
\text { DTL, EXO1, HIF1A, KIF20A, LOXL2, MIF, NASP, } \\
\text { NFYA, OLR1, PXN, PCNA, PSMB9, RFC3, STAT1, } \\
\text { SSH1, SNAPC1, SNRPF, SNRPG, TBL1XR1, ZNF367 }\end{array}$ & 43 & $1.40 E-04$ \\
\hline & GO:0070062 & $\begin{array}{l}\text { Extracellular } \\
\text { exosome }\end{array}$ & $\begin{array}{l}\text { ATP6V1C1, BAX, CD276, CD44, CDC42BPA, } \\
\text { GNA13, H2AFZ, LYN, AP2M1, AK2, B2M, CA2, } \\
\text { CLIC4, DST, FTH1, FN1, FLNA, HPRT1, IGFBP3, } \\
\text { ITGB1, IL1B, MIF, MSN, MYO1B, MYO5A, MYH9, } \\
\text { MTMR11, NRAS, OLR1, PLSCR1, PLAT, PLAU, } \\
\text { PCNA, PSMB9, SPP1, SERPINE1, SLC7A5, SLC7A8, } \\
\text { SOD2, THBS1, TGM2, TMEM33, TPM3 }\end{array}$ & 43 & $1.80 E-04$ \\
\hline & GO:0016020 & Membrane & $\begin{array}{l}\text { ATP2B4, BAX, DDX18, POLD1, ERCC6L, GNA13, } \\
\text { HTATIP2, KRAS, LARP4, RECQL, TTK, AGPS, } \\
\text { ASPH, B2M, CAV1, CAV2, CAPRIN1, CENPE, CEP55, } \\
\text { FLNA, HIP1, ITGB1, LOXL2, MYO5A, MYH9, NRAS, } \\
\text { NRP2, NDE1, OLR1, PLSCR1, PGF, PHLDA2, } \\
\text { SLC7A11, SLC7A5, SLC7A8 }\end{array}$ & 35 & $4.70 E-04$ \\
\hline
\end{tabular}


TABLE 4: Continued.

\begin{tabular}{|c|c|c|c|c|c|}
\hline Category & & Term & Genes & Count & $P$ value \\
\hline \multirow[t]{5}{*}{$\begin{array}{l}\text { Molecular } \\
\text { function }\end{array}$} & GO:0005515 & Protein binding & $\begin{array}{l}\text { ABL2, ATP6V1C1, ATP2B4, BAX, BCL2L11, } \\
\text { CD276, CD44, CDC42BPA, DDX18, POLD1, DSCC1, } \\
\text { E2F3, ERCC6L, FOSL1, GNA13, GABPB1, GINS2, } \\
\text { GINS4, H2AFZ, HTATIP2, KRAS, LYN, MET, NMI, } \\
\text { NUDCD1, OIP5, PDLIM7, POP1, PPFIA1, RAD54B, } \\
\text { RAD54L, RECQL, SHC1, TTK, WDHD1, YEATS2, } \\
\text { AP2M1, ADM, AGPS, APOL1, ASPH, BIRC5, B2M, } \\
\text { CASK, CA2, CSNK2A1, CAV1, CAV2, CDCA3, } \\
\text { CENPE, CENPL, CEP55, CLIC4, COL4A1, CCNA2, } \\
\text { CCNE2, CDKN3, DTL, DCBLD2, DST, ECE2, EXO1, } \\
\text { FTH1, FN1, FLNA, GLS, HIP1, HPRT1, HIF1A, IGFBP3, } \\
\text { ITGB1, IL1A, IL24, KIF20A, LOXL2, MIF, MAGOHB, } \\
\text { MSN, MYH9, NASP, NRIP3, NFYA, NDE1, OLR1, PXN, } \\
\text { PLSCR1, PGF, PLAT, PLAU, PHLDB2, PCNA, PSMB9, } \\
\text { RFC3, SPP1, SCG5, SERPINE1, STAT1, SSH1, SNRPF, } \\
\text { SNRPG, SLC7A11, SLC7A8, THBS1, TBL1XR1, TACC3, } \\
\text { TGM2, TMEM33, TCOF1, TROAP, TPM3 }\end{array}$ & 110 & $5.30 E-07$ \\
\hline & GO:0005524 & ATP binding & $\begin{array}{l}\text { ABL2, ATP2B4, CDC42BPA, DDX18, ERCC6L, KRAS, } \\
\text { LYN, MET, RAD54B, RAD54L, RECQL, TTK, AK2, } \\
\text { CASK, CSNK2A1, CENPE, DYRK3, DNAH17, GMPS, } \\
\text { KIF20A, MYO1B, MYO5A, MYH9, TGM2 }\end{array}$ & 24 & $7.70 E-03$ \\
\hline & GO:0042802 & $\begin{array}{l}\text { Identical protein } \\
\text { binding }\end{array}$ & $\begin{array}{c}\text { BAX, CDC42BPA, NMI, BIRC5, B2M, CAV1, FN1, } \\
\text { HPRT1, NDE1, PCNA, STAT1, SOD2, THBS1 }\end{array}$ & 13 & $3.80 E-02$ \\
\hline & GO:0005102 & Receptor binding & $\begin{array}{c}\text { ABL2, CD276, LYN, ADM, CAV1, GRP, MIF, MSN, } \\
\text { PLAT, SERPINE1, TNFSF4 }\end{array}$ & 11 & $1.40 E-03$ \\
\hline & GO:0046982 & $\begin{array}{l}\text { Protein } \\
\text { heterodimerization } \\
\text { activity }\end{array}$ & $\begin{array}{c}\text { BAX, GABPB1, H2AFZ, BIRC5, CAV1, CAV2, HIP1, } \\
\text { HIF1A, ITGB1, PGF }\end{array}$ & 10 & $2.50 E-02$ \\
\hline \multirow{5}{*}{$\begin{array}{l}\text { KEGG } \\
\text { PATHWAY }\end{array}$} & hsa05200 & Pathways in cancer & $\begin{array}{c}\text { BAX, E2F3, GNA13, KRAS, MET, BIRC5, COL4A1, } \\
\text { CCNE2, IL24, FN1, HIF1A, ITGB4, MMP1, NRAS, } \\
\text { PGF, STAT1, TPM3 }\end{array}$ & 17 & $5.90 E-05$ \\
\hline & hsa05205 & Proteoglycans in cancer & $\begin{array}{l}\text { CD44, KRAS, MET, CAV1, CAV2, FN1, FLNA, HIF1A, } \\
\text { ITGB1, MSN, NRAS, PXN, PLAU, THBS1 }\end{array}$ & 14 & $6.30 E-07$ \\
\hline & hsa04151 & $\begin{array}{l}\text { PI3K-Akt signaling } \\
\text { pathway }\end{array}$ & $\begin{array}{l}\text { BCL2L11, KRAS, MET, COL4A1, CCNE2, FN1, } \\
\text { ITGB1, IL2RA, NRAS, OSMR, PGF, SPP1, THBS1 }\end{array}$ & 13 & $8.10 E-04$ \\
\hline & hsa04510 & Focal adhesion & $\begin{array}{l}\text { MET, SHC1, CAV1, CAV2, COL4A1, FN1, FLNA, } \\
\text { ITGB1, PXN, PGF, SPP1, THBS1 }\end{array}$ & 12 & $3.20 E-05$ \\
\hline & hsa05206 & MicroRNAs in cancer & $\begin{array}{l}\text { BCL2L11, CD44, E2F3, KRAS, MET, SHC1, CCNE2, } \\
\text { GLS, NRAS, PLAU, THBS1 }\end{array}$ & 11 & $2.20 E-03$ \\
\hline
\end{tabular}

${ }^{\mathrm{b}}$ KEGG: Kyoto Encyclopedia of Genes and Genomes.

\section{Discussion}

The downregulation of miR-1-3p in HNSCC was supported by 1,140 HNSCC and 243 noncancer tissue samples from TCGA, GEO, Oncomine, ArrayExpress, and SRA databases, which enhanced the dependability of our results. miR-1-3p's low expression could be associated with the burgeoning of HNSCC. To study the functional implication of miRNA-1 in HNSCC cells and identify new neoplastic paths, a more reliable set of target genes was obtained by integrating potential target genes composed of four parts: miR-1-transfected DEGs, sequence DEGs, chip DEGs, and the targets in the prediction tool. KEGG pathway analysis showed the most significant pathways were "the pathways in cancer," "proteoglycans in cancer," "PI3K-Akt signaling pathway," "focal adhesion," and "MicroRNAs in cancer." We confirmed pivotal targets of miR-1-3p. ITGB4 was one of the most important targets.

In recent years, research on noncoding RNAs appears to have advanced rapidly, especially the inquiry of miRNAs. A large number of miRNAs exert their functions in human diseases [26-28]. Most miRNAs serve as tumor suppressors for human cancers, such as miR-874 [29, 30], miR$21[31,32]$, and miR-155 [33], while some miRNAs are overexpressed in human cancer tissues, such as miR-93, miR-218, and miR-375. Likewise, many miRNAs regulate miRNA expression and promote or suppress HNSCC cell 


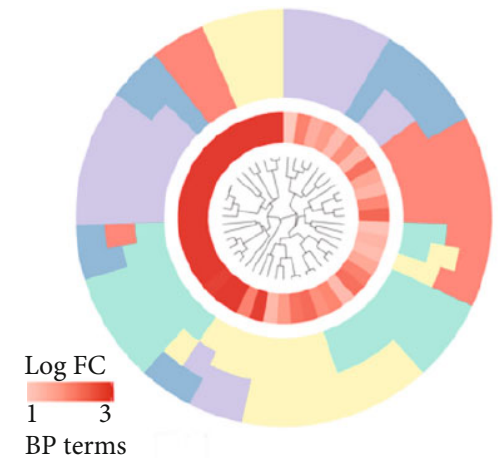

$\square$ Leukocyte migration

$\square$ Apoptotic process

$\square$ Positive regulation of cell proliferation

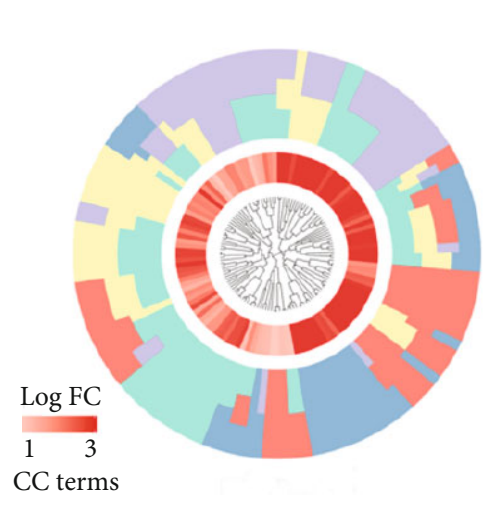

$\square$ Cytoplasm

$\square$ Extracellular exosome

$\square$ Cytosol

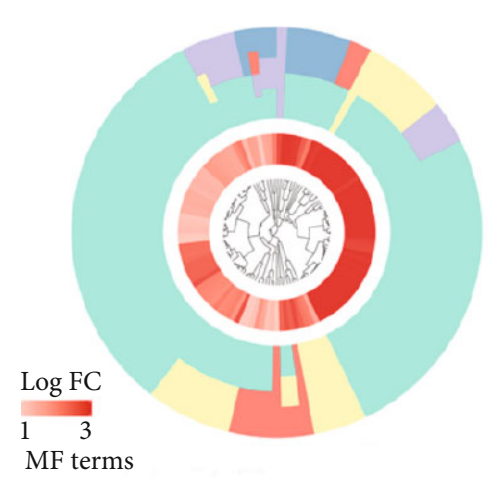

Protein binding

$\square$ Receptor binding

$\square$ ATP binding
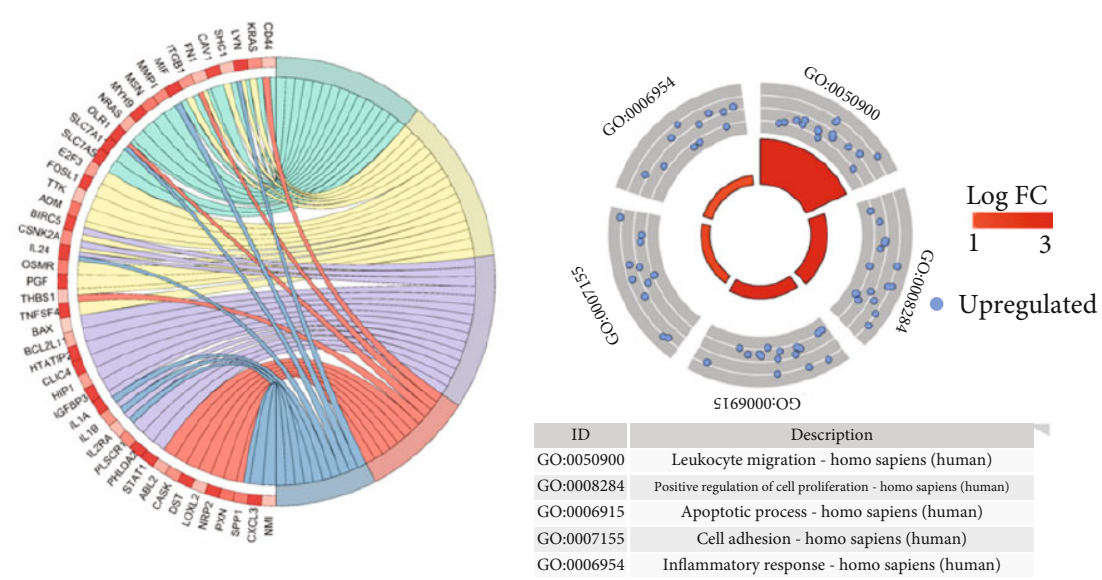

$\square$ Cell adhesion

$\square$ Inflammatory response

(a)
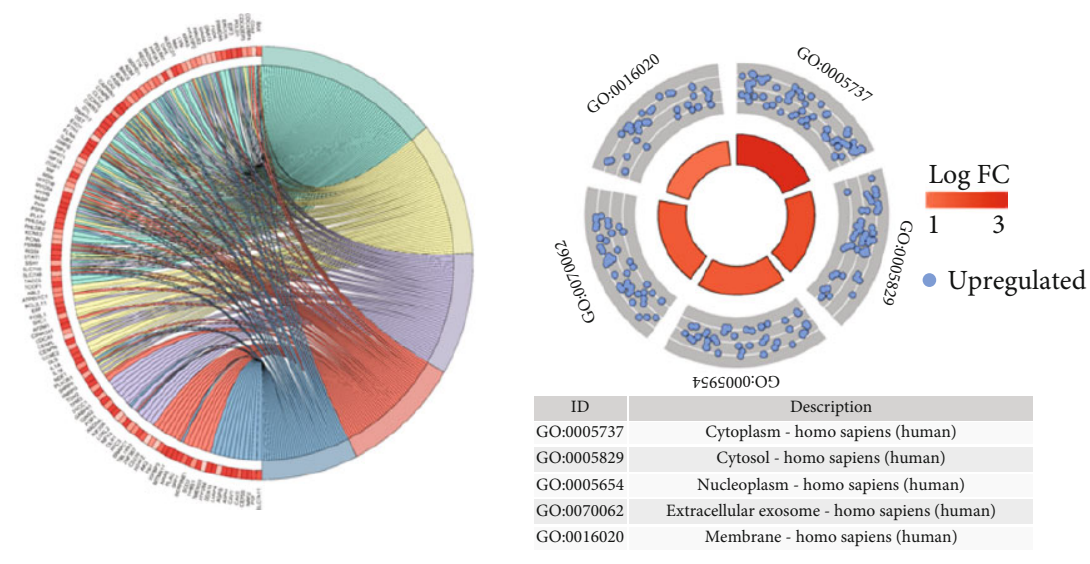

Nucleoplasm

$\square$ Membrane

(b)
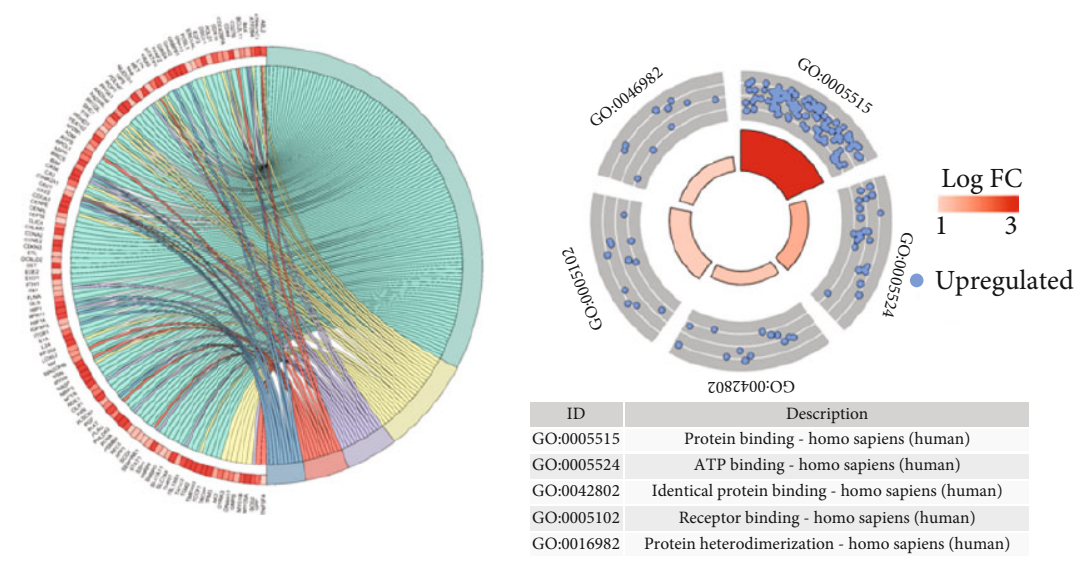

$\square$ Protein heterodimerization activity

$\square$ Identical protein binding

(c)

FIgUre 7: Continued. 


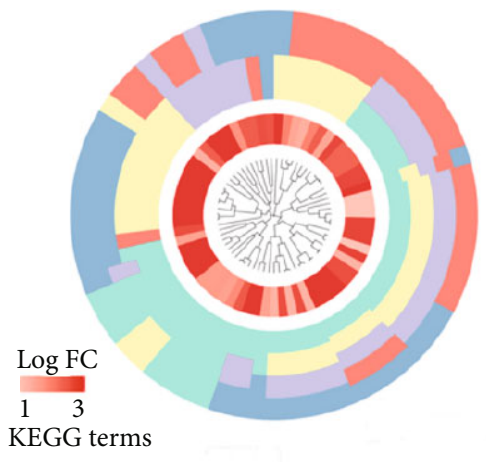

Pathways in cancer

Focal adhesion

Proteoglycans in cancer
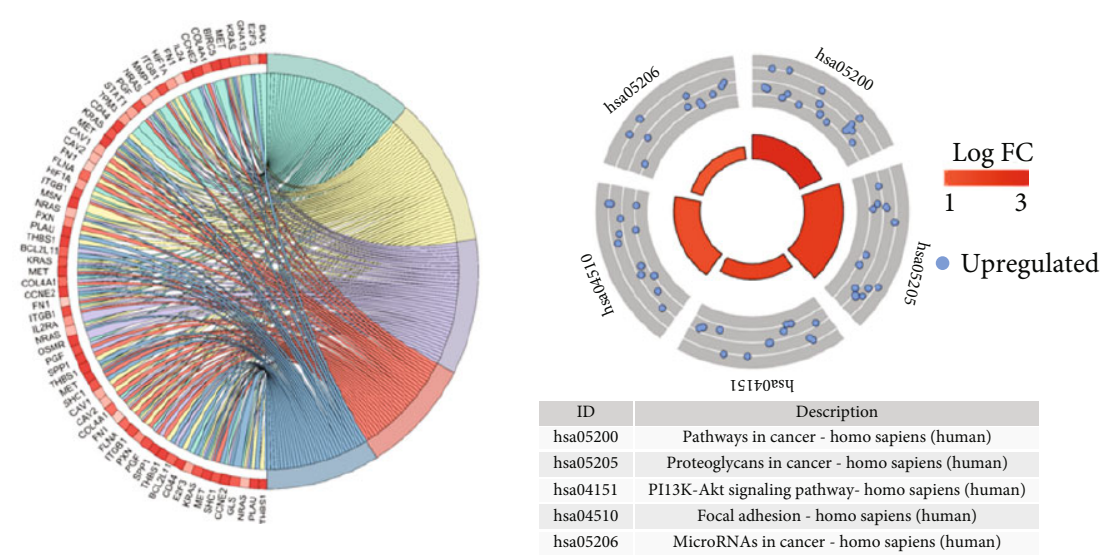

MicroRNAs in cancer

PI3K-Akt signaling pathway

(d)

FIGURE 7: Gene enrichment circles of miR-1-3p in HNSCC: (a) biological process; (b) cellular component; (c) molecular function; (d) KEGG pathways.

proliferation [1, 34]. miR-1-3p was proven dysregulated in HNSCC [16, 35]. Nohata et al. [15] demonstrated that miR-1 was downregulated in HNSCC samples and disclosed that transgelin 2 (TAGLN2) was directly adjusted by miR-1. Koshizuka et al. [1] verified gene expression in HNSCC and found that miR-1 was reduced clearly in HNSCC tissues. However, only eight studies have investigated miR-1 expression in HNSCC. Further studies are needed to determine the relationship between miR-1 and HNSCC.

The 174 upregulated differentially expressed genes were screened from 39 datasets, three miR-1 transfection samples, and a prediction tool using the $\mathrm{R}$ language tool. Although the gene list was not large, screening was rigorous. The obtained difference genes were accurate and had a certain degree of persuasion. The KEGG enrichment pathway is involved in multifarious cancer processes. G proteincoupled receptors (GPCRs) play a vital role in signal transmission [36, 37]. Targeted proteins recognize and bind to binding sites in eukaryotes and activate a series of signaling pathways, causing changes in tumor cell states, promoting tumor blood vessel regeneration, and participating in the occurrence and course of neoplasms [38-41]. Therefore, understanding the specific mechanism of GPCR involvement in malignant tumors and related target genes is conducive to providing new opportunities for cancer prevention and treatment $[36,37]$. Some studies have explored this pathway in bladder cancer [42, 43], colorectal cancer [38], melanoma [44, 45], endometrial cancer [46], lung cancer [47], renal cell carcinoma [48, 49], and thyroid cancer [50, 51]. Moreover, Koshizuka et al. found that miR-1 inhibited tumor growth by targeting growth factor receptors and participated in various signaling pathways, including the "pathways in cancer," which was consistent with our results [1].

As key genes corresponding to important action sites, which contained important signal pathway information, we focused on hub genes in further research. Eleven hub genes were enriched in KEGG pathways, most of which had been reported by a large number of previous studies on HNSCC. ITGB4 caught our attention. The results showed that ITGB4 was highly expressed in HNSCC and was harmful to patient prognosis $(P<0.001)$.

Integrin family, a family of cell adhesion receptors, is recognized to play a key role in malignant tumor metastasis [52]. As a component of the basement membrane, the expression levels of laminin-5 and its ligand were negatively correlated with tumor invasiveness, metastasis, and poor clinical prognosis [53-55]. ITGB4 encodes a receptor for laminin-5. Studies have shown that the decreased expression of ITGB4 and laminin-5 genes occurs during the progression of prostate intraepithelial neoplasia and the development of prostate cancer [56]. Meanwhile, ITGB4 can be used as a target site to form a lump in colorectal cancer [57], gastric cancer [58, 59], prostate cancer [60-62], lung cancer [63], and other diseases $[64,65]$ to regulate the progress of diseases. A single study explored the extracellular matrix- (ECM-) receptor interaction, and ITGB4 can be an underlying target for the diagnosis and treatment of HNSCC [66]. Through PCR analysis of oral squamous cell carcinoma data and assessment of pathological clinical parameters, Nagata et al. [67] found that ITGB4 could promote distant metastasis of tumors. ITGB4 is a good biological indicator of tumors. In this study, we used bioinformatics methods to speculate that ITGB4 gene can influence the disease course of HNSCC, and ITGB4 gene was correlated with miR-1-3p. In the treatment of HNSCC, this feature could be used to develop an inhibitor of ITGB4 for treatment of HNSCC.

Our research aimed to integrate microarrays and miRNA sequencing to study the expression and deep- 


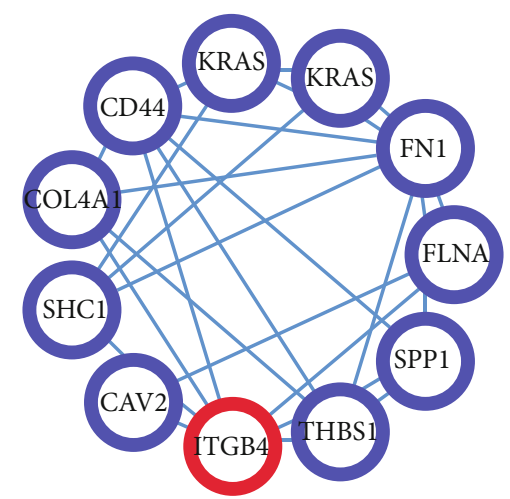

(a)

Profiled in mutations

Profiled in putative copy-number alterations from GISTIC

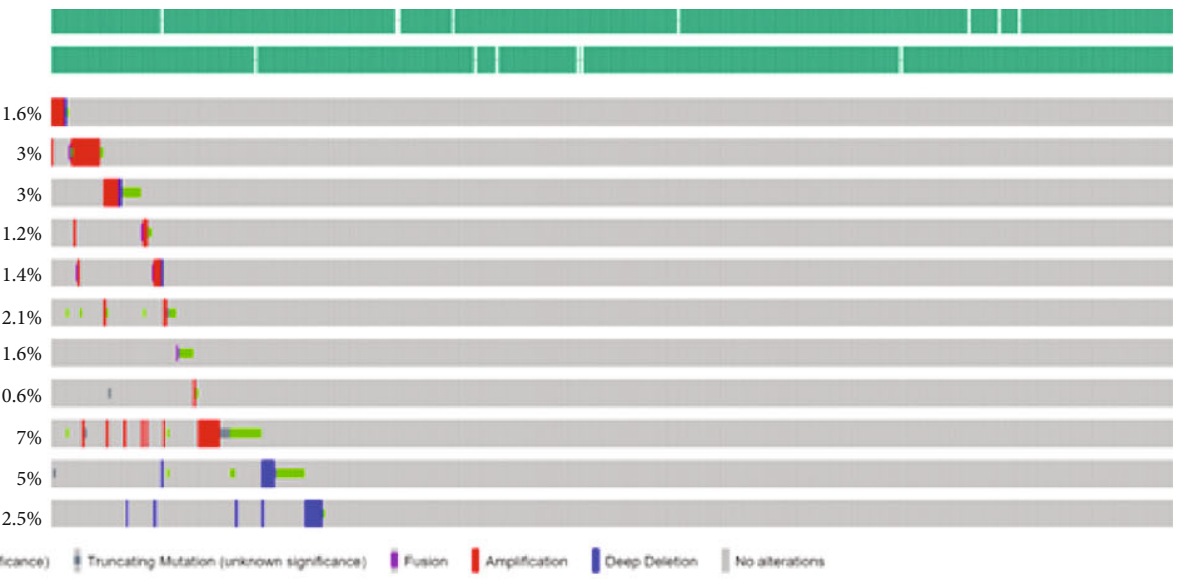

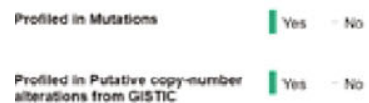

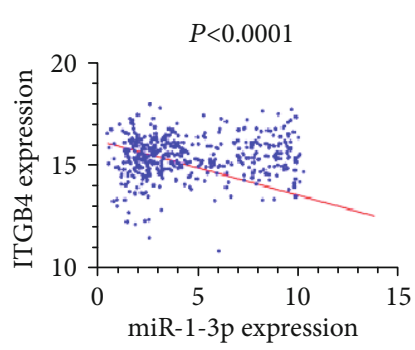

(c)

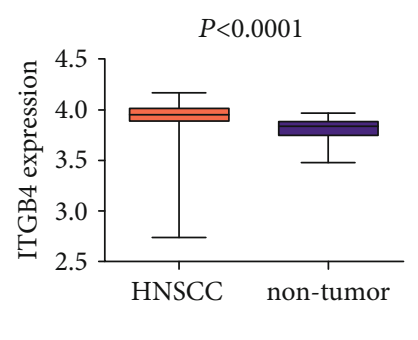

(d)

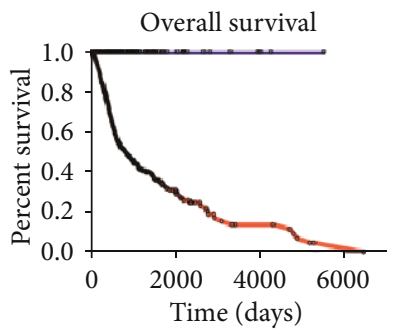

- ITGB4 low expression

- ITGB4 high expression

(e)

FIGURE 8: Preliminary prediction of ITGB4 as target gene of miR-1-3p. (a) Protein interaction network of 11 hub proteins. (b) 11 proteins were genetically altered in the HNSCC based on cBioPortal website. (c) Pearson correlation analysis showed that miR-1-3p was negatively correlated with ITGB4 $(P<0.0001)$. (d) ITGB4 expressed highly in HNSCC tumor tissues than in noncancer tissues. (e) Kaplan-Meier survival curve was used to analyze the ITGB4 expression data and evaluate its effects on the prognosis of HNSCC. ITGB4 had an apparent influence on the survival of HNSCC patients $(P<0.0001)$.

seated mechanism of miR-1-3p in HNSCC. There are limitations in this research. First, data came from online databases; thus, larger clinical samples are needed for further experimental inquiry. Second, the targeting relationship between miR-1-3p and ITGB4 was preliminarily verified, but further experiments are needed before clinical application.

\section{Conclusion}

Our research confirms the downregulation of miR-1-3p in HNSCC, revealing that miR-1-3p can act on target genes, activate signaling pathways, and participate in the development of HNSCC. ITGB4 may be a novel biological target protein, which requires further experimentation. 


\section{Data Availability}

The datasets generated and analyzed in the present study are available from the corresponding author upon reasonable request.

\section{Conflicts of Interest}

The authors declare that they have no conflicts of interest.

\section{Acknowledgments}

This work was supported by the Innovation Project of Guangxi Graduate Education (YCSW2020115).

\section{References}

[1] K. Koshizuka, T. Hanazawa, I. Fukumoto et al., "Dual-receptor (EGFR and c-MET) inhibition by tumor-suppressive _miR-1_ and _miR-206_ in head and neck squamous cell carcinoma," Journal of Human Genetics, vol. 62, no. 1, pp. 113-121, 2017.

[2] S. Perdomo, P. H. Avogbe, M. Foll et al., "Circulating tumor DNA detection in head and neck cancer: evaluation of two different detection approaches," Oncotarget, vol. 8, no. 42, pp. 72621-72632, 2017.

[3] V. Pozzi, D. Sartini, R. Rocchetti et al., "Identification and characterization of cancer stem cells from head and neck squamous cell carcinoma cell lines," Cellular Physiology and Biochemistry: International Journal of Experimental Cellular Physiology, Biochemistry, and Pharmacology, vol. 36, no. 2, pp. 784-798, 2015.

[4] A. Jou and J. Hess, "Epidemiology and molecular biology of head and neck cancer," Oncology research and treatment, vol. 40, no. 6, pp. 328-332, 2017.

[5] A. R. Krishnan, H. Zheng, J. G. Kwok et al., "A comprehensive study of smoking-specific microRNA alterations in head and neck squamous cell carcinoma," Oral Oncology, vol. 72, pp. 56-64, 2017.

[6] K. Koshizuka, T. Hanazawa, I. Fukumoto, N. Kikkawa, Y. Okamoto, and N. Seki, "The microRNA signatures: aberrantly expressed microRNAs in head and neck squamous cell carcinoma," Journal of Human Genetics, vol. 62, no. 1, pp. 313, 2017.

[7] C. Han, Y. Zhou, Q. An et al., "MicroRNA-1 (miR-1) inhibits gastric cancer cell proliferation and migration by targeting MET," Tumour biology: the journal of the International Society for Oncodevelopmental Biology and Medicine, vol. 36, no. 9, pp. 6715-6723, 2015.

[8] X. Wu, S. Li, X. Xu et al., "The potential value of miR-1 and miR-374b as biomarkers for colorectal cancer," International Journal of Clinical and Experimental Pathology, vol. 8, no. 3, pp. 2840-2851, 2015.

[9] R. Liu, J. Li, Y. Lai, Y. Liao, R. Liu, and W. Qiu, "Hsa-miR-1 suppresses breast cancer development by down-regulating Kras and long non-coding RNA MALAT1," International Journal of Biological Macromolecules, vol. 81, pp. 491-497, 2015.

[10] L. Wu, T. Wang, D. He, X. Li, and Y. Jiang, "miR-1 inhibits the proliferation of breast cancer stem cells by targeting EVI-1," OncoTargets and therapy, vol. Volume 11, pp. 8773-8781, 2018.

[11] L. Gao, P. Yan, F. F. Guo, H. J. Liu, and Z. F. Zhao, "MiR-1-3p inhibits cell proliferation and invasion by regulating BDNF-
TrkB signaling pathway in bladder cancer," Neoplasma, vol. 65 , no. 1, pp. 89-96, 2018.

[12] J. Zhang, L. Wang, S. Mao et al., "miR-1-3p contributes to cell proliferation and invasion by targeting glutaminase in bladder cancer cells," Cellular physiology and biochemistry: international journal of experimental cellular physiology, biochemistry, and pharmacology, vol. 51, no. 2, pp. 513-527, 2018.

[13] T. Hu, Y. F. Chang, Xiao et al., "miR-1 inhibits progression of high-risk papillomavirus-associated human cervical cancer by targeting G6PD," Oncotarget, vol. 7, no. 52, pp. 86103-86116, 2016.

[14] F. An, Z. Zhang, M. Xia, and L. Xing, "Subpath analysis of each subtype of head and neck cancer based on the regulatory relationship between miRNAs and biological pathways," Oncology Reports, vol. 34, no. 4, pp. 1745-1754, 2015.

[15] N. Nohata, Y. Sone, T. Hanazawa et al., "miR-1 as a tumor suppressive microRNA targeting TAGLN2 in head and neck squamous cell carcinoma," Oncotarget, vol. 2, no. 1-2, pp. 29-42, 2011.

[16] X. Chen, J. Shi, J. Zhong et al., "miR-1, regulated by LMP1, suppresses tumour growth and metastasis by targeting K-ras in nasopharyngeal carcinoma," International Journal of Experimental Pathology, vol. 96, no. 6, pp. 427-432, 2015.

[17] C. Jin, B. Yan, Q. Lu, Y. Lin, and L. Ma, "The role of MALAT1/miR-1/slug axis on radioresistance in nasopharyngeal carcinoma," Tumour biology: the journal of the International Society for Oncodevelopmental Biology and Medicine, vol. 37, no. 3, pp. 4025-4033, 2016.

[18] J. Lu, F. P. Zhao, Z. Peng et al., "EZH2 promotes angiogenesis through inhibition of miR-1/Endothelin-1 axis in nasopharyngeal carcinoma," Oncotarget, vol. 5, no. 22, pp. 11319-11332, 2014.

[19] C. D. Wu, Y. S. Kuo, H. C. Wu, and C. T. Lin, "MicroRNA-1 induces apoptosis by targeting prothymosin alpha in nasopharyngeal carcinoma cells," Journal of Biomedical Science, vol. 18, p. 80, 2011.

[20] Z. Wang, J. Wang, Z. Chen, K. Wang, and L. Shi, "MicroRNA1-3p inhibits the proliferation and migration of oral squamous cell carcinoma cells by targeting DKK1," Biochemistry and cell biology = Biochimie et biologie cellulaire, vol. 96, no. 3, pp. 355364, 2018.

[21] A. Colaprico, T. C. Silva, C. Olsen et al., "TCGAbiolinks: an $\mathrm{R} /$ Bioconductor package for integrative analysis of TCGA data," Nucleic Acids Research, vol. 44, no. 8, article e71, 2016.

[22] T. Barrett, S. E. Wilhite, P. Ledoux et al., "NCBI GEO: archive for functional genomics data sets-update," Nucleic Acids Research, vol. 41, no. D1, pp. D991-D995, 2012.

[23] N. Kolesnikov, E. Hastings, M. Keays et al., "ArrayExpress update-simplifying data submissions," Nucleic Acids Research, vol. 43, no. D1, pp. D1113-D1116, 2015.

[24] D. R. Rhodes, J. Yu, K. Shanker et al., “_ONCOMINE_ : A Cancer Microarray Database and Integrated Data-Mining Platform," Neoplasia, vol. 6, no. 1, pp. 1-6, 2004.

[25] R. Leinonen, H. Sugawara, M. Shumway, and on behalf of the International Nucleotide Sequence Database Collaboration, "The sequence read archive," Nucleic Acids Research, vol. 39, no. Database, pp. D19-D21, 2011.

[26] N. Li, X. Pan, J. Zhang et al., "Plasma levels of miR-137 and miR-124 are associated with Parkinson's disease but not with Parkinson's disease with depression," Neurological sciences: official journal of the Italian Neurological Society and of the 
Italian Society of Clinical Neurophysiology, vol. 38, no. 5, pp. 761-767, 2017.

[27] X. Meng, V. Müller, K. Milde-Langosch, F. Trillsch, K. Pantel, and $\mathrm{H}$. Schwarzenbach, "Diagnostic and prognostic relevance of circulating exosomal miR-373, miR-200a, miR-200b and miR-200c in patients with epithelial ovarian cancer," Oncotarget, vol. 7, no. 13, pp. 16923-16935, 2016.

[28] A. M. Maged, W. S. Deeb, A. el Amir et al., "Diagnostic accuracy of serum miR-122 and miR-199a in women with endometriosis," International Journal of Gynaecology and Obstetrics: The Official Organ of the International Federation of Gynaecology and Obstetrics, vol. 141, no. 1, pp. 14-19, 2018.

[29] B. Xia, M. Lin, W. Dong et al., "Upregulation of miR-874-3p and miR-874-5p inhibits epithelial ovarian cancer malignancy via SIK2," Journal of Biochemical and Molecular Toxicology, vol. 32, no. 8, article e22168, 2018.

[30] T. Jiang, L.-Y. Guan, Y.-S. Ye, H.-Y. Liu, and R. Li, "MiR-874 inhibits metastasis and epithelial-mesenchymal transition in hepatocellular carcinoma by targeting $<\mathrm{i}>\mathrm{SOX} 12</ \mathrm{i}\rangle$," American Journal of Cancer Research, vol. 7, no. 6, pp. 1310-1321, 2017.

[31] M. S. Masoudi, E. Mehrabian, and H. Mirzaei, "MiR-21: a key player in glioblastoma pathogenesis," Journal of Cellular Biochemistry, vol. 119, no. 2, pp. 1285-1290, 2018.

[32] P. Wang, Q. Guan, D. Zhou, Z. Yu, Y. Song, and W. Qiu, "miR21 inhibitors modulate biological functions of gastric cancer cells via PTEN/PI3K/mTOR pathway," DNA and Cell Biology, vol. 37, no. 1, pp. 38-45, 2018.

[33] J. Zuo, Y. Yu, M. Zhu et al., "Inhibition of miR-155, a therapeutic target for breast cancer, prevented in cancer stem cell formation," Cancer biomarkers: section A of Disease markers, vol. 21, no. 2, pp. 383-392, 2018.

[34] N. Kikkawa, T. Kinoshita, N. Nohata et al., "microRNA-504 inhibits cancer cell proliferation via targeting CDK6 in hypopharyngeal squamous cell carcinoma," International Journal of Oncology, vol. 44, no. 6, pp. 2085-2092, 2014.

[35] Z. Liao, X. Wang, H. Liang et al., "miR-1 suppresses the proliferation and promotes the apoptosis of esophageal carcinoma cells by targeting Src," Cancer Medicine, vol. 6, no. 12, pp. 2957-2965, 2017.

[36] M. O’Hayre, M. S. Degese, and J. S. Gutkind, "Novel insights into $G$ protein and $G$ protein-coupled receptor signaling in cancer," Current Opinion in Cell Biology, vol. 27, pp. 126135, 2014.

[37] R. Lappano and M. Maggiolini, "G protein-coupled receptors: novel targets for drug discovery in cancer," Nature Reviews. Drug Discovery, vol. 10, no. 1, pp. 47-60, 2011.

[38] T. Takayama, K. Miyanishi, T. Hayashi, Y. Sato, and Y. Niitsu, "Colorectal cancer: genetics of development and metastasis," Journal of Gastroenterology, vol. 41, no. 3, pp. 185-192, 2006.

[39] M. Takata and T. Saida, "Genetic alterations in melanocytic tumors," Journal of Dermatological Science, vol. 43, no. 1, pp. 1-10, 2006.

[40] A. D. Panani and C. Roussos, "Cytogenetic and molecular aspects of lung cancer," Cancer Letters, vol. 239, no. 1, pp. 19, 2006.

[41] F. Amant, P. Moerman, P. Neven, D. Timmerman, E. van Limbergen, and I. Vergote, "Endometrial cancer," The Lancet, vol. 366, no. 9484, pp. 491-505, 2005.

[42] A. P. Mitra, R. H. Datar, and R. J. Cote, "Molecular pathways in invasive bladder cancer: new insights into mechanisms, pro- gression, and target identification," Journal of Clinical Oncology, vol. 24, no. 35, pp. 5552-5564, 2006.

[43] K. Sugano and T. Kakizoe, "Genetic alterations in bladder cancer and their clinical applications in molecular tumor staging," Nature Clinical Practice. Urology, vol. 3, no. 12, pp. 642-652, 2006.

[44] C. Levy, M. Khaled, and D. E. Fisher, "MITF: master regulator of melanocyte development and melanoma oncogene," Trends in Molecular Medicine, vol. 12, no. 9, pp. 406-414, 2006.

[45] A. J. Miller and M. C. Mihm, "Melanoma," The New England Journal of Medicine, vol. 355, no. 1, pp. 51-65, 2006.

[46] J. L. Hecht and G. L. Mutter, "Molecular and pathologic aspects of endometrial carcinogenesis," Journal of Clinical Oncology, vol. 24, no. 29, pp. 4783-4791, 2006.

[47] S. Aviel-Ronen, F. H. Blackhall, F. A. Shepherd, and M. S. Tsao, "K-ras mutations in non-small-cell lung carcinoma: a review," Clinical Lung Cancer, vol. 8, pp. 30-38, 2006.

[48] S. Sudarshan, P. A. Pinto, L. Neckers, and W. M. Linehan, "Mechanisms of disease: hereditary leiomyomatosis and renal cell cancer-a distinct form of hereditary kidney cancer," Nature Clinical Practice. Urology, vol. 4, no. 2, pp. 104-110, 2007.

[49] S. Sudarshan, W. M. Linehan, and L. Neckers, "HIF and fumarate hydratase in renal cancer," British Journal of Cancer, vol. 96, no. 3, pp. 403-407, 2007.

[50] M. Santoro, R. M. Melillo, and A. Fusco, "RET/PTC activation in papillary thyroid carcinoma: European Journal of Endocrinology prize lecture," European Journal of Endocrinology, vol. 155, no. 5, pp. 645-653, 2006.

[51] H. V. Reddi, B. McIver, S. K. Grebe, and N. L. Eberhardt, "The paired box-8/peroxisome proliferator-activated receptorgamma oncogene in thyroid tumorigenesis," Endocrinology, vol. 148, no. 3, pp. 932-935, 2007.

[52] S. J. Moschos, L. M. Drogowski, S. L. Reppert, and J. M. Kirkwood, "Integrins and cancer," Oncology, vol. 21, 9 Supplement 3, pp. 13-20, 2007.

[53] R. L. Stewart and K. L. O'Connor, "Clinical significance of the integrin _ $\alpha_{-} 6 \_\beta_{-} 4$ in human malignancies," Laboratory Investigation, vol. 95, no. 9, pp. 976-986, 2015.

[54] J. Banyard, I. Chung, M. Migliozzi et al., "Identification of genes regulating migration and invasion using a new model of metastatic prostate cancer," BMC Cancer, vol. 14, no. 1, pp. 387-387, 2014.

[55] M. Wang, R. B. Nagle, B. S. Knudsen, G. C. Rogers, and A. E. Cress, "A basal cell defect promotes budding of prostatic intraepithelial neoplasia," Journal of Cell Science, vol. 130, no. 1, pp. 104-110, 2017.

[56] T. L. Davis, A. E. Cress, B. L. Dalkin, and R. B. Nagle, "Unique expression pattern of the alpha6beta4 integrin and laminin-5 in human prostate carcinoma," Prostate, vol. 46, no. 3, pp. 240-248, 2001.

[57] A. Ferraro, C. K. Kontos, T. Boni et al., "Epigenetic regulation of miR-21 in colorectal cancer," Epigenetics, vol. 9, no. 1, pp. 129-141, 2014.

[58] D. Hong, X. Zhang, R. Li et al., "Deletion of _TMEM268_ inhibits growth of gastric cancer cells by downregulating the ITGB4 signaling pathway," Cell Death and Differentiation, vol. 26, no. 8, pp. 1453-1466, 2019.

[59] Z.-Y. Yang, H. Jiang, Y. Qu et al., "Metallopanstimulin-1 regulates invasion and migration of gastric cancer cells partially 
through integrin $\beta 4$," Carcinogenesis, vol. 34, no. 12, pp. 28512860, 2013.

[60] J. Yao, C. Xu, Z. Fang et al., “Androgen receptor regulated microRNA miR-182-5p promotes prostate cancer progression by targeting the ARRDC3/ITGB4 pathway," Biochemical and Biophysical Research Communications, vol. 474, no. 1 , pp. 213-219, 2016.

[61] K. Kawakami, Y. Fujita, T. Kato et al., "Integrin $\beta 4$ and vinculin contained in exosomes are potential markers for progression of prostate cancer associated with taxane-resistance," International Journal of Oncology, vol. 47, no. 1, pp. 384-390, 2015.

[62] I. Eke, A. Y. Makinde, M. J. Aryankalayil et al., "Long-term tumor adaptation after radiotherapy: therapeutic implications for targeting integrins in prostate cancer," Molecular cancer research: MCR, vol. 16, no. 12, pp. 1855-1864, 2018.

[63] W. Zheng, C. Jiang, and R. Li, "Integrin and gene network analysis reveals that ITGA5 and ITGB1 are prognostic in non-small-cell lung cancer," OncoTargets and Therapy, vol. 9, pp. 2317-2327, 2016.

[64] M. Fanelli, A. Camperchioli, L. Petrella et al., "Non-catalytic region of tyrosine kinase adaptor protein 2 (NCK2) pathways as factor promoting aggressiveness in ovarian cancer," The International journal of biological markers, vol. 33, no. 1 , pp. 124-131, 2018.

[65] C. Leng, Z. G. Zhang, W. X. Chen et al., "An integrin beta4EGFR unit promotes hepatocellular carcinoma lung metastases by enhancing anchorage independence through activation of FAK-AKT pathway," Cancer Letters, vol. 376, no. 1, pp. 188-196, 2016.

[66] B. Yang, Z. Chen, Y. Huang, G. Han, and W. Li, "Identification of potential biomarkers and analysis of prognostic values in head and neck squamous cell carcinoma by bioinformatics analysis," OncoTargets and therapy, vol. Volume 10, pp. 2315-2321, 2017.

[67] M. Nagata, A. A. Noman, K. Suzuki et al., "ITGA3 and ITGB4 expression biomarkers estimate the risks of locoregional and hematogenous dissemination of oral squamous cell carcinoma," BMC Cancer, vol. 13, no. 1, 2013. 